\title{
NESTED HOARE TRIPLES AND FRAME RULES FOR HIGHER-ORDER STORE*
}

\author{
JAN SCHWINGHAMMER $^{a}$, LARS BIRKEDAL $^{b}$, BERNHARD REUS $^{c}$, AND HONGSEOK YAN $^{d} \mathrm{G}$ \\ ${ }^{a}$ Programming Systems Lab, Saarland University, 66123 Saarbrücken, Germany \\ e-mail address: jan@ps.uni-saarland.de \\ ${ }^{b}$ IT University of Copenhagen, Rued Langgaards Vej 7, 2300 København S., Denmark \\ e-mail address: birkedal@itu.dk \\ ${ }^{c}$ School of Informatics, University of Sussex, Brighton BN1 9QH, U.K. \\ e-mail address: bernhard@sussex.ac.uk \\ ${ }^{d}$ Department of Computer Science, University of Oxford, Oxford OX1 3QD, U.K. \\ e-mail address: Hongseok.Yang@cs.ox.ac.uk
}

\begin{abstract}
Separation logic is a Hoare-style logic for reasoning about programs with heap-allocated mutable data structures. As a step toward extending separation logic to high-level languages with ML-style general (higher-order) storage, we investigate the compatibility of nested Hoare triples with several variations of higher-order frame rules.

The interaction of nested triples and frame rules can be subtle, and the inclusion of certain frame rules is in fact unsound. A particular combination of rules can be shown consistent by means of a Kripke model where worlds live in a recursively defined ultrametric space. The resulting logic allows us to elegantly prove programs involving stored code. In particular, using recursively defined assertions, it leads to natural specifications and proofs of invariants required for dealing with recursion through the store.
\end{abstract}

\section{INTRODUCTION}

Many programming languages permit not only the storage of first-order data, but also forms of higher-order store. Examples are code pointers in C, and ML-like general references. It is therefore important to have modular reasoning principles for these language features. Separation logic is an effective formalism for modular reasoning about pointer programs, in low-level C-like programming languages and, more recently, also in higher-level languages [13, 14, 17, 25]. However, its assertions are usually limited to talk about first-order data.

In previous work, we have begun the study of separation logic for languages with higherorder store [5, 23]. A challenge in this research is the combination of proof rules from separation logic for modular reasoning, and proof rules for code stored on the heap. Ideally,

1998 ACM Subject Classification: F.3.1, F.3.2.

Key words and phrases: Higher-order store, Hoare logic, separation logic, semantics.

* A preliminary version of this work was presented at the 18th EACSL Annual Conference on Computer Science Logic (CSL'09), 7-11 September 2009, Coimbra, Portugal [26]. 
a program logic for higher-order store provides sufficiently expressive proof rules that, e.g., can deal with recursion through the store, and at the same time interact well with (higherorder) frame rules, which enable modular program verification.

Our earlier work [5, 23] shows that separation logic is consistent with higher-order store. However, the formulation in this earlier work has a shortcoming: code is treated like any other data in that assertions can only mention concrete commands. In order to obtain modular, open and reusable reasoning principles, it is clearly desirable to abstract from particular code and instead (partially) specify its behaviour. For example, when verifying mutually recursive procedures on the heap, one would like to consider each procedure in isolation, relying on properties but not the implementations of the others. The recursion rule given by Birkedal et al. [5] and Reus and Schwinghammer [23] does not achieve this. A second, and less obvious consequence of lacking behavioural specifications for code in assertions is that one cannot take full advantage of the frame rules of separation logic. For instance, the programming language in [5] can simulate higher-order procedures by passing arguments through the heap, but the available (higher-order) frame rules are not useful here because an appropriate specification for this encoding is missing.

In this article, we address these shortcomings by investigating a program logic in which stored code can be specified using Hoare triples, i.e., an assertion language with nested triples. This is an obvious idea, but the combination of nested triples and frame rules turns out to be tricky: the most natural combination is in fact unsound.

The main technical contributions of this article are therefore:

(1) the observation that certain "deep" frame rules can be unsound,

(2) the suggestion of a "good" combination of nested Hoare triples and frame rules, and

(3) the verification of those rules by means of an elegant Kripke model, based on a denotational semantics of the programming language, where the worlds are themselves world-dependent sets of heaps.

The worlds form a complete metric space and (the denotation of) the operation $\otimes$, needed to generically express higher-order frame rules, is contractive; as a consequence, our logic permits recursively defined assertions.

Outline. After introducing the syntax of programming language and assertions in Section 2 we discuss some unsound combinations of rules in Section 3. This section also contains the suggested set of rules for our logic. The soundness of the logic is then shown in Section 4 . Section 5 discusses further proof rules for nested triples. Finally the conclusion addresses related work and the differences between the model presented here and a step-indexed model.

\section{Syntax of Programs and Assertions}

This section presents the syntax of the programming language and that of assertions.

2.1. Programming language. We consider a simple imperative programming language extended with operations for stored code and heap manipulation. The syntax of the language is shown in Figure 1. The expressions in the language are integer expressions, variables, and the quote expression ' $C$ ' for representing an unevaluated command $C$. The integer or code value denoted by expression $e_{1}$ can be stored in a heap cell $e_{0}$ using $\left[e_{0}\right]:=e_{1}$, and this stored value can later be looked up and bound to the (immutable) variable $y$ by 


\begin{tabular}{|c|c|}
\hline $\begin{array}{c}e \in \operatorname{Exp}::=0|-1| 1|\ldots| e_{1}+e_{2}|\ldots| x \\
\mid{ }^{\prime} C\end{array}$ & $\begin{array}{l}\text { integer expressions, variable } \\
\text { quote (command as expression) }\end{array}$ \\
\hline $\begin{aligned} C \in \operatorname{Com}::= & {\left[e_{1}\right]:=e_{2} \mid \text { let } y=[e] \text { in } C \mid \text { eval }[e] } \\
\mid & \text { let } x=\text { new }\left(e_{1}, \ldots, e_{n}\right) \text { in } C \mid \text { free } e \\
& \text { skip } \mid C_{1} ; C_{2} \\
\mid & \text { if }\left(e_{1}=e_{2}\right) \text { then } C_{1} \text { else } C_{2}\end{aligned}$ & $\begin{array}{l}\text { assignment, lookup, unquote } \\
\text { allocation, disposal } \\
\text { no op, sequencing } \\
\text { conditional }\end{array}$ \\
\hline $\begin{aligned} & P, Q \in \text { Assn }::= \text { false } \mid \text { true }|P \vee Q| P \wedge Q \mid P \Rightarrow Q \\
& \mid \forall x . P|\exists x . P| e_{1}=e_{2} \mid e_{1} \leq e_{2} \\
& \mid e_{1} \mapsto e_{2}|e m p| P * Q \\
&\{P\} e\{Q\} \mid P \otimes Q \\
&|X(\vec{e})|(\mu X(\vec{x}) . P)(\vec{e}) \mid \ldots\end{aligned}$ & $\begin{array}{l}\text { intuitionistic-logic connectives } \\
\text { quantifiers, atomic formulas } \\
\text { separating connectives } \\
\text { Hoare triple, invariant extension } \\
\text { relation variable, recursion }\end{array}$ \\
\hline
\end{tabular}

FiguRE 1. Syntax of expressions, commands and assertions

let $y=\left[e_{0}\right]$ in $D$. In case the value stored in cell $e_{0}$ is code ' $C$ ', we can run (or "evaluate") this code by executing eval $\left[e_{0}\right]$. Our language also provides constructs for allocating and disposing heap cells such as $e_{0}$ above.

We point out that, as in ML, all variables $x, y, z$ in our language are immutable, so that once they are bound to a value, their values do not change. This property of the language lets us avoid side conditions on variables when studying frame rules. Finally, we do not include while loops in our language; these could be added easily, and they can also be expressed by stored code (using Landin's knot) 1

Example 2.1 (Iterate procedure). An iterator that calls its parameter function as well as itself through the store can be programmed as follows.

$$
\begin{aligned}
C_{i t, f, c} \equiv & \text { let } n=[c] \text { in } \\
& \text { if } n=0 \text { then skip else (eval }[f] ;[c]:=n-1 ; \text { eval }[i t])
\end{aligned}
$$

Here we assume that cells it, $f$ and $c$ are some fixed global constants, and that the iterator code is stored in the cell it. Command $C_{i t, f, c}$ then calls the code in $f$ as many times as the value of counter cell $c$ prescribes.

2.2. Assertions and distribution axioms. Our assertion language is standard first-order intuitionistic logic, extended with separating connectives emp and $*$, the points-to predicate $\mapsto 25$, and recursively defined assertions $(\mu X(\vec{x}) \cdot P)(\vec{e})$. The syntax of assertions appears in Figure 1. Each assertion describes a property of states, which consist of an immutable stack and a mutable heap. Formula emp means that the heap component of the state is empty, and $P * Q$ means that the heap component can be split into two, one satisfying $P$ and the other satisfying $Q$, both evaluated with respect to the same stack. The spatial implication operator ("magic wand") is omitted here for reasons explained later in Remark 4.9, The points-to predicate $e_{0} \mapsto e_{1}$ states that the heap component consists of only one cell $e_{0}$ whose content is $e_{1}$ or, in case $e_{1}$ is a command, an approximation $e^{\prime}$ of $e_{1}$ which is defined

\footnotetext{
${ }^{1}$ To obtain the original while rule of Hoare logic one needs to be able to hide the additional pointer storing the body of the while loop. This can be achieved using anti-frame rules as discussed e.g. in [28].
} 


$$
\begin{aligned}
& P \circ R \stackrel{\text { def }}{=}(P \otimes R) * R \\
& \{P\} \text { e }\{Q\} \otimes R \Leftrightarrow\{P \circ R\} \text { e }\{Q \circ R\} \\
& \left(P \otimes R^{\prime}\right) \otimes R \Leftrightarrow P \otimes\left(R^{\prime} \circ R\right) \\
& (\kappa x . P) \otimes R \Leftrightarrow \kappa x .(P \otimes R) \quad(\kappa \in\{\forall, \exists\}, x \notin f v(R)) \\
& (P \oplus Q) \otimes R \Leftrightarrow(P \otimes R) \oplus(Q \otimes R) \quad(\oplus \in\{\Rightarrow, \wedge, \vee, *\}) \\
& P \otimes R \Leftrightarrow P \quad \text { ( } P \text { is one of true, false, emp, } e=e^{\prime}, e \mapsto e^{\prime} \text { ) }
\end{aligned}
$$

Figure 2. Axioms for distributing $-\otimes R$

(terminates) for less heaps than $e_{1}$. This is in line with the fact that we consider partial correctness only.

One interesting aspect of our assertion language is that it includes Hoare triples $\{P\} e\{Q\}$ and invariant extensions $P \otimes Q$; previous work [7, 5] does not treat them as assertions but as so-called specifications, which form a different syntactic category. A consequence of having these new constructs as assertions is that they allow us to study proof rules for exploiting locality of stored code systematically, as we will describe shortly.

Intuitively, $\{P\}$ e $\{Q\}$ means that $e$ denotes code satisfying $\{P\}-\{Q\}$, and $P \otimes Q$ denotes a modification of $P$ where all the pre- and post-conditions of triples inside $P$ are $*$-extended with $Q$. In other words, all code specified by pre- and postconditions inside $P$ must preserve invariant $Q$. For instance, the assertion $(\exists k .(1 \mapsto k) \wedge\{e m p\} k\{e m p\}) \otimes(2 \mapsto 0)$ is equivalent to $(\exists k .(1 \mapsto k) \wedge\{2 \mapsto 0\} k\{2 \mapsto 0\})$. This assertion says that cell 1 is the only cell in the heap and it stores code $k$ that satisfies the triple $\{2 \mapsto 0\}-\{2 \mapsto 0\}$. This intuition about the $\otimes$ operator is made precise in the set of axioms in Figure 2, which let us distribute $\otimes$ through the constructs of the assertion language.

Note that since triples are assertions, they can appear in pre- and post-conditions of triples. This nested use of triples is useful in reasoning, because it allows one to specify stored code behaviourally, in terms of properties that it satisfies. Typically, a program logic consists of both an assertion logic and a specification logic (e.g. [24]). With the introduction of nested triples, assertions and specifications necessarily become mutually recursive; for simplicity, we have chosen to identify our specification and assertion logics and just work with a single logic of assertions.

A second interesting aspect of our assertion language is that assertions include $(n$ ary) relation variables $X(\vec{e})$, and that assertions can be defined recursively: the assertion $(\mu X(\vec{x}) . P)(\vec{e})$ binds $X$ and $\vec{x}=x_{1} \ldots x_{n}$ in $P$ and satisfies the axiom

$$
(\mu X(\vec{x}) \cdot P)(\vec{e}) \Leftrightarrow P[X:=\mu X(\vec{x}) \cdot P, \vec{x}:=\vec{e}] .
$$

In the case where $X$ has arity 0 we will simply write $X$ in place of $X()$.

Example 2.2 (Specification of the iterator via recursion through the store). The previously given command

$$
\begin{aligned}
C_{i t, f, c} \equiv & \text { let } n=[c] \text { in } \\
& \text { if } n=0 \text { then skip else (eval }[f] ;[c]:=n-1 ; \text { eval }[i t])
\end{aligned}
$$

can be specified as follows, if we assume that the called procedure in $f$ does preserve some invariant $I$ that does not access the counter and iterator cells $c$ and $i t$, respectively. For instance, $I$ could be emp (in case $f$ has no side effects) or $\exists m . x \mapsto m * y \mapsto n ! / m$ ! when the factorial of $n$ is computed in $y$. If $x$ contains the content of the counter then, like with 
a while loop, upon termination $y$ contains the expected result. In the following, to keep the triples simple, we assume that $I=e m p$.

$$
\left\{c \mapsto_{-} * f \mapsto\{e m p\}_{-}\{e m p\} * R_{i t}\right\}^{\prime} C_{i t, f, c}\left\{c \mapsto 0 * f \mapsto\{e m p\}_{-}\{e m p\} * R_{i t}\right\} .
$$

Here, we use the abbreviation $e \mapsto\{P\}_{-}\{Q\}$ for $(\exists k .(e \mapsto k) \wedge\{P\} k\{Q\})$, and $R_{i t}$ is a recursive specification for the iterator itself:

$$
R_{i t} \equiv \mu X . i t \mapsto\left\{c \mapsto_{-} * f \mapsto\{e m p\}_{-}\{e m p\} * X\right\}_{-}\left\{c \mapsto 0 * f \mapsto\{e m p\}_{-}\{e m p\} * X\right\} .
$$

Consequently, heap $i t \mapsto{ }^{\text {' }} C_{i t, f, c}$ ' is in $R_{i t}$ and thus one can prove (see Example 3.3) that

$$
\begin{aligned}
& \left\{c \mapsto_{-} * f \mapsto\{e m p\}_{-}\{e m p\} * i t \mapsto_{-}\right\} \\
& {[i t]:={ }^{'} C_{i t, f, c} \text { ' } \text { eval }[i t]} \\
& \left\{c \mapsto 0 * f \mapsto\{e m p\}_{-}\{e m p\} * R_{i t}\right\} .
\end{aligned}
$$

The specification for the iterator in it is recursive since the iterator calls itself through the store and any recursive call through the store requires the same specification as the original call. Assuming that procedure $f$ has no side effect, we guarantee that the iterator will have no other side effect than setting the counter to 0 . The iterator specification also works with more sophisticated behaviour of $f$ : in Example 3.2 below we will discuss how to deal with situations where $f$ has side effects on some heap space (but preserves an invariant $I$ ). It will turn out that we can generalise from invariant emp to $I$ without even having to reprove the original side-effect free specification given here, using the so-called deep frame rule.

Analogously to the definition of equi-recursive types in typed lambda calculi, for the assertion $(\mu X(\vec{x}) . P)(\vec{e})$ to be well-formed we require that $P$ is (formally) contractive in $X$ [18. This means that $X$ can occur in $P$ only in subterms of the form $\left\{P^{\prime}\right\} e\left\{Q^{\prime}\right\}$ or $P^{\prime \prime} \otimes R^{\prime}$ where $P^{\prime \prime}$ is formally contractive in $X$. (We omit the straightforward inductive definition of formal contractiveness.) Semantically, this requirement ensures that $\mu X(\vec{x}) . P$ is well-defined as a unique fixed point. Note that in particular all assertions of the form $P \otimes X$ and $\left\{P^{\prime} * X\right\} e\left\{Q^{\prime} * X\right\}$ are formally contractive in $X$, provided $X$ does not appear in $P$. Thus, $\mu X . P \otimes X$, and $\mu X$.it $\mapsto\{P * X\} e\{Q * X\}$ are well-formed (in particular, $R_{i t}$ above). Let $R$ abbreviate the latter assertion. Then, with the help of Axiom 2.1 and the distribution axioms of Figure 2 one can show that $R$ is equivalent to $i t \mapsto\{P * R\} e\{Q * R\}$ which in turn is equivalent to $i t \mapsto\{P * i t \mapsto\{P * R\} e\{Q * R\}\}$ e $\{Q * i t \mapsto\{P * R\} e\{Q * R\}\}$ and one can keep unfolding $R$ as many times as one wishes. A successful invocation of the code in it thus requires a heap satisfying $P$ as well as containing it again pointing to code that satisfies the very same specification. It is this potentially infinite unfolding that frees one from having to prove triples by various forms of induction on the number of recursive calls as in [12, 5].

More generally, in order to deal with mutually recursive stored procedures we may need to compute fixpoints of mutually recursively defined assertions. For brevity we omit formal syntax for mutual recursion. We will say more about the use of recursively defined predicates and their existence in Sections 3 and 4 . In particular, the semantics in Section 4 can be used to interpret mutually recursive families of assertions.

Finally, note that we have not included an axiom for distributing $\otimes$ through a recursive type in Figure 2, In particular, the axiom $(\mu X . P) \otimes R \Leftrightarrow \mu X .(P \otimes R)$ does not hold in the presence of nested triples. Instead, one has to use the axiom $\mu X . P \Leftrightarrow P[X:=\mu X . P]$ and unfold the recursive type to exhibit a "proper" connective through which $\otimes R$ can be distributed. 
We shall make use of two abbreviations. The first is $Q \circ R$, which stands for $(Q \otimes$ $R) * R$ and which has already been used in Figure 2, This abbreviation describes the combination of two invariants $Q$ and $R$ into a single invariant in the axiom $(P \otimes Q) \otimes R \Leftrightarrow$ $P \otimes(Q \circ R)$. It is also used to add an invariant $R$ to a Hoare triple $\{P\} e\{Q\}$, so as to obtain $\{P \circ R\} e\{Q \circ R\}$. We use the asymmetric $\circ$ instead of the symmetric $*$ here to extend not only $Q$ ( $P$ and $Q$ resp.) by $R$ but also ensure, via $\otimes$, that all Hoare triples nested inside $Q$ ( $P$ and $Q$, resp.) preserve $R$ as an invariant. The o operator has been introduced in [20], where it is credited to Paul-André Melliès and Nicolas Tabareau. The second abbreviation is for the points-to operator of separation logic: $e_{1} \mapsto P\left[e_{2}\right] \stackrel{\text { def }}{=} e_{1} \mapsto e_{2} \wedge P\left[e_{2}\right]$ and $e_{1} \mapsto P[-] \stackrel{\text { def }}{=} \exists x . e_{1} \mapsto P[x]$. Here $x$ is a fresh (logic) variable and $P[\cdot]$ is an assertion with an expression hole, such as $\{Q\} \cdot\{R\}, \cdot=e$ or $\cdot \leq e$

\section{Proof Rules for Higher-order Store}

In our formal setting, reasoning about programs is done by deriving judgements of the form $\Xi ; \Gamma \vdash P$, where $P$ is an assertion expressing properties of programs, $\Xi$ is a list of (distinct) relation variables $X_{1}, \ldots, X_{n}$ containing all the free relation variables in $P$, and $\Gamma$ is a list of (distinct) variables $x_{1}, \ldots, x_{n}$ containing all the free variables in $P$. For instance, to prove that command $C$ stores at cell 1 the code that initializes cell 10 to 0 , we need to derive $\left.\Xi ; \Gamma \vdash\left\{1 \mapsto_{-}\right\}^{\prime} C^{\prime}\left\{1 \mapsto\left\{10 \mapsto_{-}\right\}\right\}_{-}\{10 \mapsto 0\}\right\}$. (One concrete example of such a command $C$ is $[1]:={ }^{6}[10]:=0 '$.) Below, we will sometimes omit the contexts $\Xi$ and $\Gamma$ when they are empty.

In this section, we describe inference rules and axioms for assertions that let one efficiently reason about programs. We focus on those related to higher-order store.

3.1. Standard proof rules. The proof rules include the standard proof rules for intuitionistis 3 logic and the logic of bunched implications [15] (not repeated here). Moreover, the proof rules include variations of standard separation logic proof rules, see Figures 3 and 4. The (Update), (FreE) and (SkIP) rules in the figure are not the usual small axioms in separation logic, since they contain an assertion $P$ that describes the unchanged part. Since we have the standard frame rule for $*$, we could have used small axioms instead here. We chose not to do this, because the current non-small axioms make it easier to follow our discussions on frame rules and higher-order store in the next subsection. We added a specific version of (UPDATE), called (UPDATEINV), which will turn out not to be derivable from (UPDATE) because triples cannot be used in the (INVARIANCE) rule. (This will be explained in Section 5). The side condition of (INVARIANCE) " $\psi$ is pure" ensures that $\psi$ is an assertion denoting a predicate that is actually independent of the heap. Examples for pure predicates are arithmetic formulae like $x=1$.

The figure neither includes the rule for executing stored code with eval $[e]$ nor the frame rule for adding invariants to triples. The reason for this omission is that these two

\footnotetext{
${ }^{2}$ These abbreviations do not necessarily lead to a unique reading, e.g. $x \mapsto 1 \leq 2$ could mean $x \mapsto 1 \wedge 1 \leq 2$ or $x \mapsto 2 \wedge 1 \leq 2$, but we will only use them when the $P$ in question is uniquely defined.

${ }^{3} \mathrm{~A}$ classical interpretation of the assertion language is inconsistent, see Section 3.5 .
} 


$$
\begin{aligned}
& \text { DEREF } \\
& \frac{\Xi ; \Gamma, x \vdash\{P * e \mapsto x\} C^{\prime}\{Q\}}{\Xi ; \Gamma \vdash\{\exists x . P * e \mapsto x\}^{\prime} \operatorname{let} x=[e] \text { in } C^{\prime}\{Q\}}(x \notin \mathrm{fv}(e, Q))
\end{aligned}
$$

UPDATE

$$
\overline{\Xi ; \Gamma \vdash\left\{e \mapsto \mapsto_{-} * P\right\}^{\prime}[e]:=e_{0}^{\prime}\left\{e \mapsto e_{0} * P\right\}}
$$

UPDATEINV

$$
\begin{aligned}
& \overline{\Xi ; \Gamma \vdash\left\{e \mapsto{ }_{-} *\left(e_{1} \mapsto e_{0} \wedge\{A\} e_{0}\{B\}\right)\right\} '[e]:=e_{0}{ }^{\prime}\left\{\left(e \mapsto e_{0} \wedge\{A\} e_{0}\{B\}\right) *\left(e_{1} \mapsto e_{0} \wedge\{A\} e_{0}\{B\}\right)\right\}} \\
& \text { New } \\
& \frac{\Xi ; \Gamma, x \vdash\{P * x \mapsto e\}^{\prime} C^{\prime}\{Q\}}{\Xi ; \Gamma \vdash\{P\}^{\prime} \text { let } x=\text { new } e \text { in } C^{\prime}\{Q\}}(x \notin \mathrm{fv}(P, e, Q)) \\
& \text { IF } \\
& \frac{\Xi ; \Gamma \vdash\left\{P \wedge e_{0}=e_{1}\right\}^{\prime} C \text { ' }\{Q\} \quad \Xi ; \Gamma \vdash\left\{P \wedge e_{0} \neq e_{1}\right\}^{\prime} D \text { ' }\{Q\}}{\Xi ; \Gamma \vdash\{P\} \text { 'if }\left(e_{0}=e_{1}\right) \text { then } C \text { else } D \text { ' }\{Q\}} \\
& \overline{\Xi ; \Gamma \vdash\left\{e \mapsto_{-} * P\right\}^{\prime} \operatorname{free}(e)^{\prime}\{P\}} \\
& \text { SEQ } \\
& \text { SKIP } \\
& \frac{\Xi ; \Gamma \vdash\{P\}{ }^{\prime} C^{\prime}\{R\} \quad \Gamma \vdash\{R\}^{\prime} D^{\prime}\{Q\}}{\Xi ; \Gamma \vdash\{P\}^{\prime} C ; D^{\prime}\{Q\}}
\end{aligned}
$$

Figure 3. Proof rules from separation logic

rules raise nontrivial issues in the presence of higher-order store and nested triples, as we shall discuss below. We also omit the conjunction axiom for triples:

$$
\overline{\Xi ; \Gamma \vdash\left\{P_{2}\right\} e\left\{Q_{2}\right\} \wedge\left\{P_{1}\right\} e\left\{Q_{1}\right\} \Rightarrow\left\{P_{1} \wedge P_{2}\right\} e\left\{Q_{1} \wedge Q_{2}\right\}}
$$

as it is not sound (neither as a rule) in the presence of higher-order or deep frame rules, for the reasons given in [16]. If we wanted to use it we would need to restrict to precise assertions, as they do.

3.2. Proof rule for recursive assertions. Besides the axiom (2.1) which lets us unfold recursive assertions, we include a proof rule that expresses the uniqueness of recursive assertions,

$$
\begin{aligned}
& \text { RUnique } \\
& \Xi ; \Gamma \vdash R \Leftrightarrow P[X:=R] \quad \Xi ; \Gamma \vdash S \Leftrightarrow P[X:=S] \\
& \Xi ; \Gamma \vdash R \Leftrightarrow S
\end{aligned}
$$

for any $P$ formally contractive in $X$. Using this rule, the equivalence of (possibly recursively defined) assertions $R$ and $S$ can be proved by finding a suitable assertion $P$ that has both $R$ and $S$ as fixed points. 


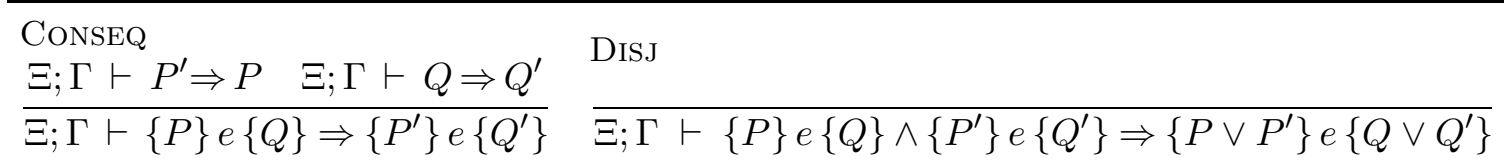

ExISTAux

$$
\overline{\Xi ; \Gamma \vdash(\forall x .\{P\} e\{Q\}) \Rightarrow\{\exists x . P\} e\{\exists x . Q\}}(x \notin \mathrm{fv}(e))
$$

INVARIANCE

$$
\overline{\Xi ; \Gamma \vdash\{P\} e\{Q\} \Rightarrow\{P \wedge \psi\} e\{Q \wedge \psi\}}(\psi \text { is pure })
$$

Figure 4. Non-syntax driven proof rules

3.3. Frame rule for higher-order store. The frame rule is the most important rule in separation logic, and it formalizes the intuition of local reasoning, where proofs focus on the footprints of the programs we verify. For instance, in Example 2.2, we have said we can prove

$$
\left\{c \mapsto_{-} * f \mapsto\{e m p\}_{-}\{e m p\} * R_{i t}\right\}^{\prime} C_{i t, f, c}{ }^{\prime}\left\{c \mapsto 0 * f \mapsto\{e m p\}_{-}\{e m p\} * R_{i t}\right\}
$$

But if we wanted now to prove a similar result for an $f$ that had some side effect like

$$
f \mapsto \text { 'let } r=[x] \text { in let } v=[y] \text { in }[y]:=r * v ;[x]:=r-1 \text { ' }
$$

then setting $I \stackrel{\text { def }}{=} \exists m . x \mapsto m * y \mapsto n ! / m$ ! we can prove $\{I\} f\{I\}$ but now we need to show

$$
\left\{c \mapsto_{-} * f \mapsto\{I\}_{-}\{I\} *\left(R_{i t} \otimes I\right) * I\right\} ' C_{i t, f, c}\left\{c \mapsto 0 * f \mapsto\{I\}_{-}\{I\} *\left(R_{i t} \otimes I\right) * I\right\}
$$

The so-called "deep frame rule" will allow us to do just that, to prove triple (3.2) from triple (3.1) in one reasoning step, such that we can re-use our original proof. This rule will be discussed below and details of its concrete usage can be seen in Example 3.2. Note also that the first-order (or shallow) frame rule does not achieve this, it would only give us

$$
\left\{c \mapsto_{-} * f \mapsto\{e m p\}_{-}\{e m p\} * R_{i t} * I\right\}{ }^{\prime} C_{i t, f, c}{ }^{\prime}\left\{c \mapsto 0 * f \mapsto\{e m p\}_{-}\{e m p\} * R_{i t} * I\right\}
$$

which is not useful here.

Establishing "deep" frame rules in our setting is challenging, because nested triples allow for several choices regarding the shape of the rule. Moreover, the recursive nature of the higher-order store complicates matters and it is difficult to see which choices actually make sense (i.e., do not lead to inconsistency).

To see this problem more clearly, consider the rules below:

$\frac{\Xi ; \Gamma \vdash\{P\} e\{Q\}}{\Xi ; \Gamma \vdash\{P \square R\} e\{Q \square R\}}$ and $\frac{}{\Xi ; \Gamma \vdash\{P\} e\{Q\} \Rightarrow\{P \square R\} e\{Q \square R\}}$ for $\square \in\{*, \circ\}$. Note that we have four choices, depending on whether we use $\square=*$ or $\square=0$ and on whether we have an inference rule or an axiom. If we choose the separating conjunction $*$ for $\square$, we obtain shallow frame rules that add $R$ to the outermost triple $\{P\} e\{Q\}$ only; they do not add $R$ in nested triples appearing in pre-condition $P$ and post-condition $Q$. On the other hand, if we choose $\circ$ for $\square$, since $(A \circ R)=(A \otimes R * R)$, we obtain deep frame 
rules that add the invariant $R$ not just to the outermost triple but also to all the nested triples in $P$ and $Q$.

The distinction between inference rule and axiom has some bearing on where the frame rule can be applied. With the axiom version, we can apply the frame rule not just to valid triples, but also to nested triples appearing in pre- or post-conditions which is not possible with the inference rule

Ideally, we would like to have the axiom versions of the frame rules for both the $*$ and $\circ$ connectives. Unfortunately, this is not possible for $\circ$ : adding the axiom version for $\circ$ makes our logic unsound. The source of the problem is that with the axiom version for $\circ$, one can add invariants selectively to some, but not necessarily all, nested triples. This flexibility can be abused to derive incorrect conclusions.

Concretely, with the axiom version for o (DeEpFrameAxiom) we can make the following derivation:

$$
\begin{aligned}
& \frac{\Xi ; \Gamma \vdash\{P \circ S\} e\{Q \circ S\}}{\Xi ; \Gamma \vdash\{P\} e\{Q\} \otimes S} \otimes-\mathrm{DisT}^{r} \frac{\overline{\Xi ; \Gamma \vdash\{P\} e\{Q\} \Rightarrow\{P \circ R\} e\{Q \circ R\}}}{\Xi ; \Gamma \vdash\{P\} e\{Q\} \otimes S \Rightarrow\{P \circ R\} e\{Q \circ R\} \otimes S} \otimes \text {-Mono } \\
& \frac{\Xi ; \Gamma \vdash\{P \circ R\} e\{Q \circ R\} \otimes S}{\Xi ; \Gamma \vdash\{(P \circ R) \circ S\} e\{(Q \circ R) \circ S\}} \otimes-\mathrm{DisT}^{r}
\end{aligned}
$$

Here we use the monotonicity of $-\otimes R$ in the form of rule ( $\otimes$-Mono), cf. Figure 9] in the Appendix. The steps annotated $\otimes$-Dist ${ }^{r}$ use the first equivalence $\{P\} e\{Q\} \otimes R \Leftrightarrow$ $\{P \circ R\} e\{Q \circ R\}$ of the distribution axioms for $\otimes$ in Fig. 2 (in $\Leftarrow$ and $\Rightarrow$ direction, respectively). We annotate the application of an axiom between triples with ${ }^{r}$ to indicate that we apply it actually as a rule via the application of (ModusPonens). So, for instance, $(\text { CONSEQ })^{r}$, used frequently below, denotes a sub-derivation of the following form:

$$
\frac{\{A\} e\{B\} \quad \frac{A^{\prime} \Rightarrow A \quad B \Rightarrow B^{\prime}}{\{A\} e\{B\} \Rightarrow\left\{A^{\prime}\right\} e\left\{B^{\prime}\right\}} \text { ConseQ }}{\left\{A^{\prime}\right\} e\left\{B^{\prime}\right\}} \text { ModusPonens }
$$

where we will usually omit the implications $A^{\prime} \Rightarrow A$ and $B \Rightarrow B^{\prime}$ when they are obvious from the context.

The fact we could derive $\{(P \circ R) \circ S\} e\{(Q \circ R) \circ S\}$ means that when adding $R$ to nested triples, we can skip the triples in the $S$ part of the pre- and post-conditions of $\{P \circ S\} e\{Q \circ S\}$. This flexibility leads to the unsoundness:

Proposition 3.1. Adding the axiom version (DEEPFrameAxiom) of the frame rule for $\circ$ renders our logic unsound.

Proof. Let $R$ be the recursive assertion $\mu X .\left(3 \mapsto\left\{1 \mapsto_{-}\right\}_{-}\left\{1 \mapsto_{-}\right\}\right) \otimes X$, and note that this means $R \Leftrightarrow\left(3 \mapsto\left\{1 \mapsto_{-}\right\}_{-}\left\{1 \mapsto_{-}\right\}\right) \otimes R$ holds. Then, we can derive the triple:

$$
\operatorname{ConSEQ}^{r} \frac{k \vdash\left\{2 \mapsto^{r}\left\{1 \mapsto_{-}\right\}_{-}\left\{1 \mapsto_{-}\right\} \circ R\right\} k\left\{2 \mapsto_{-} \circ R\right\}}{k \vdash\left\{2 \mapsto^{6} \mathrm{free}(-1)^{\prime} * 1 \mapsto_{-} * R\right\} k\left\{2 \mapsto_{-} * 1 \mapsto_{-} * 3 \mapsto\left\{1 \mapsto_{-} * R\right\}_{-}\left\{1 \mapsto_{-} * R\right\}\right\}}
$$

Here the first step uses the derivation above for adding invariants selectively, and the last step uses the consequence rule with the following two implications: 


$$
\begin{aligned}
& 2 \mapsto\left\{1 \mapsto_{-}\right\}_{-}\left\{1 \mapsto_{-}\right\} \circ 1 \mapsto_{-} \circ R \Longleftrightarrow 2 \mapsto\left\{1 \mapsto_{-} * 1 \mapsto_{-} * R\right\}_{-}\left\{1 \mapsto_{-} * 1 \mapsto_{-} * R\right\} * 1 \mapsto_{-} * R \\
& \Longleftrightarrow 2 \mapsto\{\text { false }\}_{-}\{\text {false }\} * 1 \mapsto_{-} * R \\
& \Longleftarrow 2 \mapsto \text { 'free }(-1)^{\prime} * 1 \mapsto_{-} * R
\end{aligned}
$$

where the second equivalence follows from the fact that $1 \mapsto_{-} * 1 \mapsto_{-} \Leftrightarrow$ false (use axioms $(\star-$ Overlap), ( $\star$ ZERo), and ( $\star$-Mono) of Separation Logic from Figure 9) with (CONSEQ), and

$$
\begin{aligned}
2 \mapsto_{-} \circ 1 \mapsto_{-} \circ R & \Longleftrightarrow 2 \mapsto_{-} * 1 \mapsto_{-} * R \\
& \Longleftrightarrow 2 \mapsto_{-} * 1 \mapsto_{-} *\left(\left(3 \mapsto\left\{1 \mapsto_{-}\right\}_{-}\left\{1 \mapsto_{-}\right\}\right) \otimes R\right) \\
& \Longleftrightarrow 2 \mapsto_{-} * 1 \mapsto_{-} * 3 \mapsto\left\{1 \mapsto_{-} * R\right\}_{-}\left\{1 \mapsto_{-} * R\right\} .
\end{aligned}
$$

in which the distribution axioms of Figure 2 are used, again in concert with (CONSEQ) and Separation Logic rules like $(\star-$ MONO).

Consider $C \equiv$ let $x=[2]$ in $[3]:=x$, i.e., the program that copies the contents from cell 2 to cell 3 . When $P[y] \equiv\left\{1 \mapsto_{-}\right\} y\left\{1 \mapsto_{-}\right\} \otimes R$ such that $R \Leftrightarrow 3 \mapsto P[-]$ holds,

$$
\begin{aligned}
& \frac{\overline{x \vdash\left\{3 \mapsto_{-} *(2 \mapsto x \wedge P[x])\right\}^{\prime}[3]:=x^{\prime}\{(3 \mapsto x \wedge P[x]) *(2 \mapsto x \wedge P[x])\}}}{x \vdash\left\{3 \mapsto_{-} *(2 \mapsto x \wedge P[x])\right\}{ }^{6}[3]:=x^{\prime}\{3 \mapsto P[-] * 2 \mapsto P[-]\}} \text { UPDATEINV }^{\text {CONSEQ }^{r}}
\end{aligned}
$$

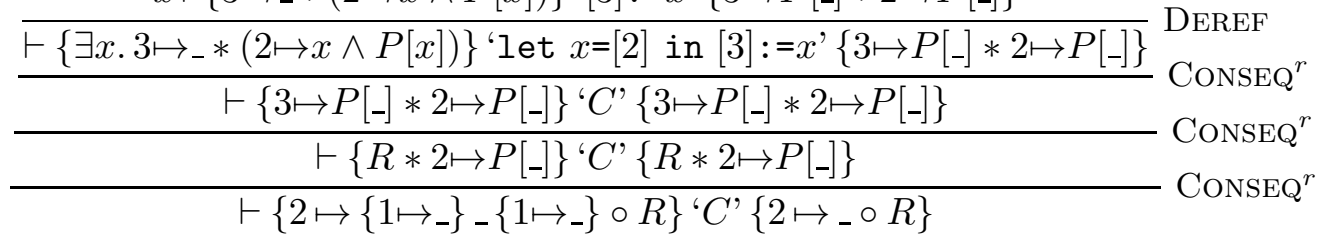

Now we instantiate $k$ in (3.4) with ' $C$ ', discharge the premise of the resulting derivation with the above derivation for $C$, and obtain

$$
\frac{\vdots}{\vdash\left\{2 \mapsto^{\prime} \operatorname{free}(-1)^{\prime} * 1 \mapsto_{-} * R\right\}^{\prime} C^{\prime}\left\{2 \mapsto_{-} * 1 \mapsto_{-} * 3 \mapsto\left\{1 \mapsto_{-} * R\right\}_{-}\left\{1 \mapsto_{-} * R\right\}\right\}}
$$

But the post-condition of the conclusion here is equivalent to $2 \mapsto_{-} * 1 \mapsto_{-} * R$ by the definition of $R$ and the distribution axioms for $\otimes$. Thus, as our rule for eval will show later, we should be able to conclude that

$$
\vdash\left\{2 \mapsto^{\prime} \operatorname{free}(-1)^{\prime} * 1 \mapsto_{-} * R\right\} \text { 'C } \text {; eval [3]' }\left\{2 \mapsto_{-} * 1 \mapsto_{-} * 3 \mapsto\left\{1 \mapsto_{-} * R\right\}_{-}\left\{1 \mapsto_{-} * R\right\}\right\}
$$

However, since -1 is not even an address, the program $(C$; eval [3]) which executes the code free(-1) now stored in cell 3 always faults, contradicting the requirement of separation logic that proved programs run without faulting.

\footnotetext{
${ }^{4}$ Note that it is important here that (CONSEQ) derives an implication between triples.
} 
Remark 3.1 (Counterexample for the Deep Frame Axiom). Notice that in the derivation above it is essential that $R$ is a recursively defined assertion, otherwise we would not obtain that the locations 2 and 3 point to code satisfying the same assertion $P$.

While the above counterexample has been the first such counterexample historically, there is also another form of counterexample discovered later which uses the same ideas as the above but works "through the store." More precisely, in this alternative counterexample the copying code ' $C$ ' resides on the heap where the frame axiom can be applied directly on a nested triple, and not through the derivation

$$
\frac{\{P \circ S\} e\{Q \circ S\}}{\{(P \circ R) \circ S\} e\{(Q \circ R) \circ S\}}
$$

This rather follows the style of $[21]^{5}$ and [10]6. For this counterexample, let $R$ be as above and let

$$
P_{1}[y] \equiv\left\{1 \mapsto_{-}\right\} y\left\{1 \mapsto_{-}\right\} .
$$

First, observe that the following triple can be derived with a rule for eval (this rule (EvAL) will be explained in detail in Section 3.4):

$$
\begin{aligned}
& \left\{2 \mapsto\{\text { false }\}_{-}\{\text {false }\} * c \mapsto\{2 \mapsto\{\text { false }\}-\{\text { false }\}\}_{-}\{2 \mapsto\{\text { false }\}-\{\text { false }\}\}\right\} \\
& \text { 'eval }[c] ' \\
& \left\{2 \mapsto \_* c \mapsto_{-}\right\}
\end{aligned}
$$

But the (DeepFrameAxiom) (the axiom version for $\circ$ ) can be used to derive

$$
c \mapsto\left\{2 \mapsto P_{1}[-]\right\}_{-}\left\{2 \mapsto P_{1}[-]\right\} \Longrightarrow c \mapsto\left\{2 \mapsto P_{1}[-] \circ\left(1 \mapsto_{-}\right)\right\}_{-}\left\{2 \mapsto P_{1}[-] \circ\left(1 \mapsto_{-}\right)\right\}
$$

which then by applying distribution axioms unfolding the definition of $P_{1}$ yields:

$$
c \mapsto\left\{2 \mapsto P_{1}[-]\right\}-\left\{2 \mapsto P_{1}[-]\right\} \Longrightarrow c \mapsto\left\{2 \mapsto\{\text { false }\}_{-}\{\text {false }\}\right\}_{-}\left\{2 \mapsto\{\text { false }\}_{-}\{\text {false }\}\right\}
$$

Applying this to triple (3.5) with the help of an appropriate $\left(\mathrm{CONSEQ}^{r}\right)$ step we can therefore derive

$$
\left.\left.\vdash\left\{2 \mapsto\{\text { false }\}_{-}\{\text {false }\} * c \mapsto\left\{2 \mapsto P_{1}[]\right]\right\}_{-}\left\{2 \mapsto P_{1}[]\right]\right\}\right\} \text { 'eval }[c] \text { ' }\left\{2 \mapsto_{-} * c \mapsto_{-}\right\}
$$

and thus by the shallow frame rule again

$$
\left.\left.\vdash\left\{1 \mapsto_{-} * 2 \mapsto\{\text { false }\}_{-}\{\text {false }\} * c \mapsto\left\{2 \mapsto P_{1}[]\right]\right\}-\left\{2 \mapsto P_{1}[]\right]\right\}\right\} \text { 'eval }[c] \text { ' }\left\{1 \mapsto_{-} * 2 \mapsto_{-} * c \mapsto_{-}\right\}
$$

This triple should not hold for all heaps since actually now the code in 2 has been laundered to work with its caller code in $c$ although the code in $c$, to function properly, might depend on the code in 2 meeting the specification $P_{1}$. Using the above derivation, we can now construct a program that is provably safe but crashes, showing that (DeEpFrameAxiom) cannot be correct (as the other used rules and axioms clearly are). First, with the rule version for $\circ$ (DEEPFRAmeRule) to add $R$ one gets

$$
\begin{aligned}
& \left\{1 \mapsto_{-} * 2 \mapsto\{\text { false }\}_{-}\{\text {false }\} * c \mapsto\left\{2 \mapsto_{1} P_{1}[]_{0} \circ R\right\}_{-}\left\{2 \mapsto P_{1}[]_{-} \circ R\right\} * R\right\} \\
& \text { 'eval }[c] \\
& \left\{1 \mapsto_{-} * 2 \mapsto_{-} * c \mapsto_{-} * R\right\}
\end{aligned}
$$

\footnotetext{
${ }^{5}$ However, the antiframe rule is used there.

${ }^{6}$ This uses a version where the copied code accesses a cell that is then disposed of before the code itself is executed later.
} 
so that by definition of $\circ, P_{1}$, and $R$ we obtain

$$
\begin{aligned}
& \left\{1 \mapsto_{-} * 2 \mapsto\{\text { false }\}_{-}\{\text {false }\} * c \mapsto\{2 \mapsto P[-] * R\}_{-}\{2 \mapsto P[] * R\} * R\right\} \\
& \text { 'eval }[c] \text { ' } \\
& \left\{1 \mapsto_{-} * 2 \mapsto_{-} * c \mapsto_{-} * R\right\}
\end{aligned}
$$

where $P[y]$ is the assertion $\left\{1 \mapsto_{-}\right\} y\left\{1 \mapsto_{-}\right\} \otimes R$ (also used in the proof of Proposition [3.1). From that one can easily derive with the rules (SEQ), (Eval) and (Conseq) that

$$
\begin{aligned}
& \left\{1 \mapsto_{-} * 2 \mapsto_{\text {ffalse }}\right\}_{-}\{\text {false }\} * c \mapsto\left\{2 \mapsto^{\prime}\left[\left[_{-}\right] * R\right\}_{-}\{2 \mapsto P[-] * R\} * R\right\} \\
& \text { 'eval }[c] \text {; eval }[3] \text { ' } \\
& \left\{1 \mapsto_{-} * 2 \mapsto_{-} * c \mapsto_{-} * R\right\}
\end{aligned}
$$

Yet, if $c \mapsto$ 'let $x=[2]$ in [3]:=x' and $2 \mapsto$ 'free $(-1)$ ', then the above program crashes. Although the code in $c$ does not call the crashing code 'free $(-1)$ ' in 2 , it copies ' $f r e e(-1)$ ' into 3, which is possible due to the "laundered" specification of 2 in the triple for $c$.

Again, this shows how essential it is that $P_{1}[-] \otimes R$ is equivalent to $R$ which forces $R$ to be recursively defined to actually allow the copying to be performed. This version of the counterexample uses the (DeepFrameRule) rather than (ModusPonens) and $(\otimes-$ MONO), and its pattern is more likely to appear in "naturally occurring" examples.

As Proposition 3.1 shows, we cannot include (DeEpFrameAxiom) in the proof system. Fortunately, the second best choice of frame axioms leads to a consistent proof system:

Proposition 3.2. Both the inference rule version of the frame rule for $\circ$ and the axiom version for $*$ are sound. In fact, the following more general version ( $\otimes$-FRAME) of the rule for $\circ$ holds:

$$
\frac{\Xi ; \Gamma \vdash P}{\Xi ; \Gamma \vdash P \otimes R}
$$

We will prove this proposition in Section 4 by a model construction.

Example 3.2 (Application of ( $\otimes$-FRAME)). Recall our specification

$$
\left\{c \mapsto_{-} * f \mapsto\{e m p\}_{-}\{e m p\} * R_{i t}\right\} \text { ' } C_{i t, f, c} \text { ' }\left\{c \mapsto 0 * f \mapsto\{e m p\}_{-}\{e m p\} * R_{i t}\right\}
$$

of the iteration command in Example 2.2. where $R_{i t}$ is a recursive specification for the iterator itself:

$$
R_{i t} \equiv \mu X . i t \mapsto\left\{c \mapsto_{-} * f \mapsto\{e m p\}_{-}\{e m p\}_{*} X\right\}_{-}\left\{c \mapsto 0 * f \mapsto\{e m p\}_{-}\{e m p\} * X\right\}
$$

Assume this triple has been already proven (cf. Example 3.3 below). If the code $C_{i t, f, c}$ is to be used on a procedure $f$ that needs some state $I$, e.g. $I \equiv a \mapsto_{-}$, then we need to show

$$
\left\{c \mapsto_{-} * f \mapsto\{I\}_{-}\{I\} *\left(R_{i t} \otimes I\right) * I\right\}^{\prime} C_{i t, f, c^{\prime}}\left\{c \mapsto 0 * f \mapsto\{I\}_{-}\{I\} *\left(R_{i t} \otimes I\right) * I\right\}
$$

This triple could be established by a proof similar to the one for the triple 3.6 above, just carrying around the extra assumption $I$. If we want to reuse this proof though, or even more importantly, if we do not have the proof of the above triple because it is part of a module for which we do not have the actual code, then we can use rule ( $\otimes$-FrAmE) on triple (3.6) to derive:

$$
\left(\left\{c \mapsto_{-} * f \mapsto\{e m p\}_{-}\{e m p\} * R_{i t}\right\}^{'} C_{i t, f, x}{ }^{\prime}\left\{c \mapsto 0 * f \mapsto\{e m p\}_{-}\{e m p\} * R_{i t}\right\}\right) \otimes I
$$


A ConseQ ${ }^{r}$ step using the equivalence of the first axiom in Figure 2 in both directions for the pre- and postcondition, respectively, thus gives us the triple:

$$
\begin{aligned}
& \left\{\left(c \mapsto_{-} \otimes I\right) *\left(f \mapsto\{e m p\}_{-}\{e m p\} \otimes I\right) *\left(R_{i t} \otimes I\right) * I\right\} \\
& \text { ' } C_{i t, f, x} \text { ' } \\
& \left\{(c \mapsto 0 \otimes I) *\left(f \mapsto\{e m p\}_{-}\{e m p\} \otimes I\right) *\left(R_{i t} \otimes I\right) * I\right\}
\end{aligned}
$$

which by another four applications of distribution axioms yields the required triple. Note that the rule (RUNIQUE) would be needed to show that $R_{i t} \otimes I$ is equivalent to the recursive assertion

$$
\mu Y . i t \mapsto\left\{c \mapsto_{-} * f \mapsto\{I\}_{-}\{I\} * I * Y\right\}_{-}\left\{c \mapsto 0 * f \mapsto\{I\}_{-}\{I\} * I * Y\right\}
$$

3.4. Rule for executing stored code. An important and challenging part of the design of a program logic for higher-order store is the design of a proof rule for eval $[e]$, the command that executes code stored at $e$. Indeed, the rule should overcome two challenges directly related to the recursive nature of higher-order store: (1) implicit recursion through the store (i.e., Landin's knot), and (2) extensional specifications of stored code.

These two challenges are addressed, using the expressiveness of our assertion language, by the following rule for eval $[e]$ :

$$
\frac{\text { EvaL }}{\Xi ; \Gamma, k \vdash R[k] \Rightarrow\{P * e \mapsto R[-]\} k\{Q\}}
$$

This rule states that in order to prove $\{P * e \mapsto R[]\}$ 'eval $[e]$ ' $\{Q\}$ for executing stored code in $[e]$ under the assumption that $e$ points to arbitrary code $k$ (expressed by the - which is an abbreviation for $\exists k . e \mapsto R[k]$ ), it suffices to show that the specification $R[k]$ implies that $k$ itself fulfils triple $\{P * e \mapsto R[]\} k\{Q\}$.

In the above rule we do not make any assumptions about what code $e$ actually points to, as long as it fulfils the specification $R$. It may even be updated between recursive calls. However, for recursion through the store, $R$ must be recursively defined as it needs to maintain itself as an invariant of the code in $e$.

Example 3.3 (Recursion through the store with the iterator). As seen in the iterator Example 2.2 one would like to prove

$$
\left\{c \mapsto_{-} * f \mapsto\{e m p\}_{-}\{e m p\} * R_{i t}\right\} \text { 'eval }[i t]^{\prime}\left\{c \mapsto 0 * f \mapsto\{e m p\}_{-}\{e m p\} * R_{i t}\right\}
$$

with the help of (EvAL). First we set

$$
R \equiv\left\{c \mapsto_{-} * f \mapsto\{e m p\}_{-}\{e m p\} * R_{i t}\right\}_{-}\left\{c \mapsto 0 * f \mapsto\{e m p\}_{-}\{e m p\} * R_{i t}\right\}
$$

such that $R_{i t}$ is the same as $i t \mapsto R[-]$. We are now in a position to apply (EvAL) obtaining the following proof obligation

$$
R[k] \Rightarrow\left\{c \mapsto_{-} * f \mapsto\{e m p\}_{-}\{e m p\} * i t \mapsto R[-]\right\} k\left\{c \mapsto 0 * f \mapsto\{e m p\}_{-}\{e m p\} * R_{i t}\right\}
$$

which can be seen to be identical to $R[k] \Rightarrow R[k]$ which holds trivially.

The (EvaL) rule crucially relies on the expressiveness of our assertion language, especially the presence of nested triples and recursive assertions. In our previous work, we did 
EvalNonRec1

$$
\overline{\Xi ; \Gamma \vdash\{P * e \mapsto \forall \vec{y} \cdot\{P\}-\{Q\}\} \text { 'eval }[e]^{\prime}\{Q * e \mapsto \forall \vec{y} \cdot\{P\}-\{Q\}\}}
$$

EVALNONRECUPD

$$
\overline{\Xi ; \Gamma \vdash\left\{P * e \mapsto \forall \vec{y} \cdot\{P * e \mapsto-\}_{-}\{Q\}\right\} \text { 'eval }[e] '\{Q\}}
$$

EvALREC

$$
\overline{\Xi ; \Gamma \vdash\{P \circ R\} \text { 'eval }[e]^{\prime}\{Q \circ R\}}\left(\text { where } R=\mu X .\left(e \mapsto \forall \vec{y} \cdot\{P\}-\{Q\} * P_{0}\right) \otimes X\right)
$$

Figure 5. Derived rules from Eval

not consider nested triples. As a result, we had to reason explicitly with stored code, rather than properties of the code, as illustrated by one of our previous rules for eval [5]:

$$
\begin{aligned}
& \text { OldEval } \\
& \frac{\Xi}{\Xi} \Gamma \vdash\{P\} \text { 'eval }[e]^{\prime}\{Q\} \Rightarrow\{P\}{ }^{\prime} C \text { ' }\{Q\} \\
& \hline \Xi ; \Gamma \vdash\left\{P * e \mapsto{ }^{\prime} C^{\prime}\right\} \text { 'eval }[e]^{\prime}\left\{Q * e \mapsto{ }^{\prime} C^{\prime}\right\}
\end{aligned}
$$

Here the actual code $C$ is specified explicitly in the pre- and post-conditions of the triple. In both rules the intuition is that the premise states that the body of the recursive procedure fulfils the triple, under the assumption that the recursive call already does so. In the (EvAL) rule this is done without direct reference to the code itself, using the variable $k$ to stand for arbitrary code satisfying $R$. The soundness proof of (OLDEvaL) proceeded along the lines of Pitts' method for establishing relational properties of domains [19]. On the other hand, as we will show in Section 4, (EVAL) relies on the availability of recursive assertions, the existence of which is guaranteed by Banach's fixpoint theorem.

From the (Eval) rule one can easily derive the axioms of Figure 5. The first two axioms are for non-recursive calls. This can be seen from the fact that in the pre-condition of the nested triples $e$ does not appear at all or does not have a specification, respectively. Only the third axiom (EvalRec) allows for recursive calls. The idea of this axiom is that one assumes that the code in $[e]$ fulfils the required triple provided the code that $e$ points to at call-time fulfils the triple as well. Let us look at the actual derivation of (EvALREC) to make this evident. We write

$$
S[k] \equiv \forall \vec{y} \cdot\{P \circ R\} k\{Q \circ R\}
$$

such that for the original

$$
R=\mu X .\left(e \mapsto \forall \vec{y} \cdot\{P\}_{-}\{Q\} * P_{0}\right) \otimes X
$$

of the rule (EvalREC) we obtain with the help of Axiom (2.1):

$$
R \Leftrightarrow(e \mapsto S[]]) *\left(P_{0} \otimes R\right)
$$


Note that in the derivation below $\Gamma$ contains the variables $\vec{y}$ which may appear freely in $P$ and $Q$.

$$
\begin{aligned}
& \frac{\Xi ; \Gamma, k \vdash(\forall \vec{y} \cdot\{P \circ R\} k\{Q \circ R\}) \Rightarrow\{P \circ R\} k\{Q \circ R\}}{\Xi ; \Gamma, k \vdash S[k] \Rightarrow\{P \circ R\} k\{Q \circ R\}} \text { FOL } \\
& \frac{\Xi ; \Gamma, k \vdash S[k] \Rightarrow\left\{(P \otimes R) * e \mapsto S[-] *\left(P_{0} \otimes R\right)\right\} k\{Q \circ R\}}{\frac{\Xi}{\Xi ; \Gamma \vdash\left\{(P \otimes R) * e \mapsto S[-] *\left(P_{0} \otimes R\right)\right\}} \text { 'eval }[e] '\{Q \circ R\}} \\
& \frac{\Xi ; \Gamma \vdash\{P \circ R\} \text { 'eval }[e]^{\prime}\{Q \circ R\}}{\text { Eval }} \\
& \text { ConsEQ }^{r}
\end{aligned}
$$

In the derivation tree above, the axiom used at the top is simply a first-order axiom for $\forall$ elimination. The quantified variables $\vec{y}$ are substituted by the variables with the same name from the context. After an application of rule (EvALREC), those variables $\vec{y}$ can then be substituted further. Step Csub abbreviates the following derivation where contexts have been omitted for clarity:

$$
\begin{aligned}
& \frac{\vdots}{S[k] \Rightarrow\{P \circ R\} k\{Q \circ R\}} \\
& \overline{P \circ R \Rightarrow P \circ R} \mathrm{R} \Rightarrow \\
& \frac{P \circ R \Rightarrow P \circ R}{(P \otimes R) * R \Rightarrow P \circ R} \text { DEF. } \circ
\end{aligned}
$$

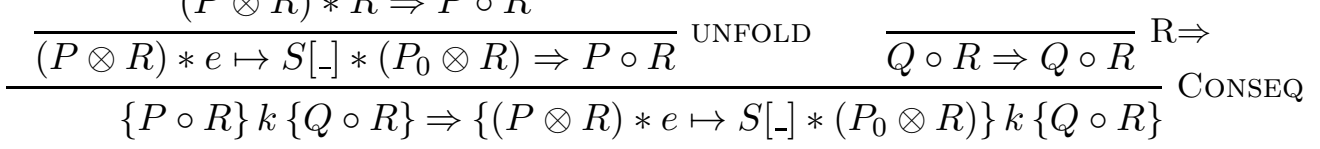

$$
\begin{aligned}
& S[k] \Rightarrow\left\{(P \otimes R) * e \mapsto S[-] *\left(P_{0} \otimes R\right)\right\} k\{Q \circ R\} \quad \mathrm{T} \Rightarrow
\end{aligned}
$$

In the above derivation, $(\mathrm{R} \Rightarrow)$ and $(\mathrm{T} \Rightarrow)$ denote reflexivity and transitivity of implication, respectively, and step UNFOLD denotes the following sub-derivation:

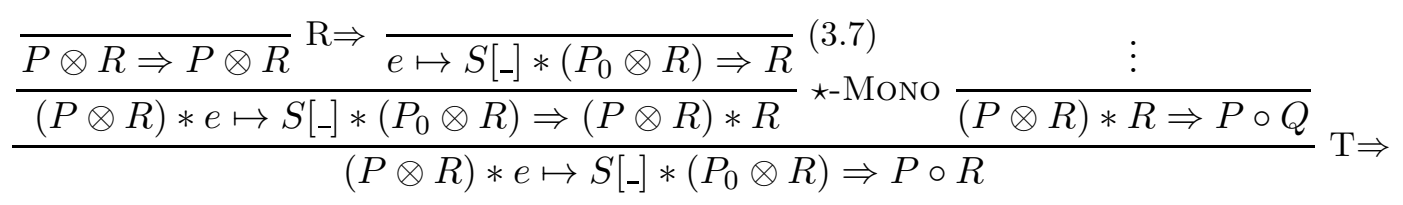

The use of recursive specification

$$
R=\mu X .\left(e \mapsto \forall \vec{y} \cdot\{P\}-\{Q\} * P_{0}\right) \otimes X
$$

is essential here as it allows us to unroll the definition (see equivalence (3.7)) so that the (EVAL) rule can be applied. Note that in the logic of [12], which also uses nested triples but features neither a specification logic nor any frame rules or axioms, recursive specifications do not exist. Avoiding them, one loses an elegant specification mechanism to allow for code updates during recursion. Such updates are indeed possible as eval uses a pointer to call code from the (obviously changeable) heap. In the logic of [12] specifications would have to refer to other means to deal with such code updates, like e.g. families of code with uniform specifications. But it is unclear to what extent such a formulation would allow for modular extensions. For modular reasoning one must not rely on concrete families of code in proofs, otherwise these proofs are not reusable when the family has to be changed to allow for 


\begin{tabular}{|c|c|c|}
\hline $\begin{array}{l}\otimes \text {-Frame } \\
\quad \Xi ; \Gamma \vdash P\end{array}$ & *-FrAme & $\begin{array}{l}\text { EvaL } \\
\Xi ; \Gamma, k \vdash R[k] \Rightarrow\{P * e \mapsto R[-]\} k\{Q\}\end{array}$ \\
\hline$\overline{\Xi ; \Gamma \vdash P \otimes R}$ & $\overline{\Xi ; \Gamma \vdash\{P\} e\{Q\} \Rightarrow\{P * R\} e\{Q * R\}}$ & $\Xi ; \Gamma \vdash\{P * e \mapsto R[-]\}$ 'eval $[e]$ ' $\{Q\}$ \\
\hline
\end{tabular}

Figure 6. Proof rules specific to higher-order store

additional code. Assuming the code in $e$ does not change, the recursively defined $R$ above can be expressed without recursion (we can omit the $P_{0}$ now, as this is only needed for mutually recursively defined triples) as follows:

$$
e \mapsto\{e \mapsto k * P\} k\{e \mapsto k * Q\}
$$

The question however remains how the assertion can be proved for some concrete ' $C$ ' that is stored in [e]. In [12] this is done by an induction on some appropriate argument, which is possible since only total correctness is considered there. In our logic, (OLDEVAL) is strikingly similar to a fixpoint induction rule in "de Bakker and Scott" style and (EvAL) even allows one to abstract away from concrete code. These rules are elegant and simple to use. Not only do they allow for recursion through the store, (EvAL) also disentangles the reasoning from the concrete code stored in the heap, supporting modularity and extensibility.

Figure 6 summarizes a particular choice of proof-rule set from the current and previous subsections. Soundness is proved in Section 4 .

3.5. Nested triples and classical assertion logic. One may wonder why we insist on an intuitionistic program logic. Unfortunately, as the following proposition shows, it is not possible to use a classical version of our logic; more precisely, the combination of a classical specification logic and rule ( $\otimes-$ FrAmE) is not sound. Thus, by our identification of assertion and specification language, we cannot have a classical assertion logic either.

Proposition 3.3. Adding rule ( $\otimes-\mathrm{FRAME})$ to a classical specification logic is not sound.

Proof. Assuming the rule for the elimination of double negation, we can derive the problematic triple

$$
\{\text { true }\} \text { 'skip' }\{\text { false }\} \text {. }
$$

Assume $\neg\{$ true $\}$ 'skip' \{false $\}$, using the abbreviation $\neg \varphi$ for $\varphi \Rightarrow$ false. With rule $(\otimes-$ FRAME) to frame in false we can derive the triple $(\neg\{$ true $\}$ 'skip' $\{$ false $\}) \otimes$ false from $\neg\{$ true $\}$ 'skip' \{false $\}$. Since true $*$ false $\Leftrightarrow$ false and false $*$ false $\Leftrightarrow$ false, rule (CONSEQ) and the distribution axioms then let us derive $\neg\{$ false $\}$ 'skip' $\{$ false $\}$. On the other hand, rule (SKIP) derives the triple $\{$ false $\}$ 'skip' $\{$ false $\}$. Thus, we have shown that from the assumption $\neg\{$ true $\}$ 'skip' $\{$ false $\}$ we can derive false, i.e. we have shown $\neg \neg\{$ true $\}$ 'skip' $\{$ false $\}$. By eliminating the double negation we can now derive the triple $\{$ true $\}$ 'skip' $\{$ false $\}$.

Note that this derivation does not use nested triples, and also applies to the specification logics used in [7, 5]. 


\section{Semantics of Nested Triples}

This section develops a model for the programming language and logic we have presented. The semantics of programs, given in Subsection 4.2 using an untyped domain-theoretic model, is standard. The following semantics of the logic is, however, unusual; it is a possible world semantics where the worlds live in a recursively defined metric space. Before we begin with the technical devlopment proper we give a brief overview of the main ideas employed.

4.1. Overview of the technical development. In earlier work, Birkedal, Torp-Smith, and Yang [7, 8] showed how to model a specification logic with higher-order frame rules but for a language with first-order store. There, the assertion and specification logic were kept distinct. Assertions were modelled as semantic predicates Pred $=P(H)$, with $H$ the set of heaps, and specifications as world-indexed truth values $W \rightarrow 2$. (These latter maps were restricted to be monotone in a certain sense, but that does not matter for the present explanation.) The informal idea was that the set of worlds would consist of invariants that had been framed in and thus worlds consisted of semantic predicates, $W=$ Pred. Here, with higher-order store and nested triples and the collapse of assertion and specification logic, assertions will be modelled as world-indexed predicates. So we get Pred $=W \rightarrow P(H)$. Worlds will still consist of semantic predicates, so $W=$ Pred. Thus we see that the set of worlds $W$ should be recursively defined. This captures the idea that any assertion can serve as an invariant to be framed in via a frame rule.

The idea of using such a Kripke model over a recursively defined set of worlds comes from [6], where this idea was used to define a model of a type system with general MLlike references (hence higher-order store). Following [6] we show how to find a solution to the recursive world equation in a category of complete bounded ultra-metric spaces (the definition of which we recall below). This is possible by restricting the subsets of $H$ that we use to so-called uniform admissible subsets of $H$. The set UAdm of all such forms a complete bounded ultra-metric space and thence we can solve the recursive world equation. Having solved that, we show how to define a world extension operator $\otimes$ (which will be used to model the syntactic $\otimes$ operator used earlier), as a fixed point of a suitable contractive operator. Moreover, we show that the subset $U A d m$ of $P(H)$ is a complete Heyting algebra with a commutative and monotone monoid structure, as needed for the interpretation of separation logic.

Having defined semantic predicates in certain metric spaces allows us to interpret recursively defined assertions via application of Banach's fixed point theorem.

The final core idea in the development is the interpretation of triples. Here we bake in the frame rules to the model by including suitable quantifications over future worlds, following ideas from earlier work [5]. To ensure that nested triples are modelled as semantic predicates, we also force the interpretation of triples to be metrically non-expansive in the worlds argument. In particular, predicates involving nested triples can be used in recursive definitions of assertions.

4.2. Semantics of expressions and commands. The interpretation of the programming language is given in the category $\mathbf{C p p o} \mathbf{p}_{\perp}$ of pointed cpos and strict continuous functions 7

\footnotetext{
${ }^{7}$ As usual, $\sqsubseteq$ denote the partial order of a cpo and $\perp$ denotes the least element of a pointed cpo, ie. $\perp \sqsubseteq d$ for any $d$.
} 


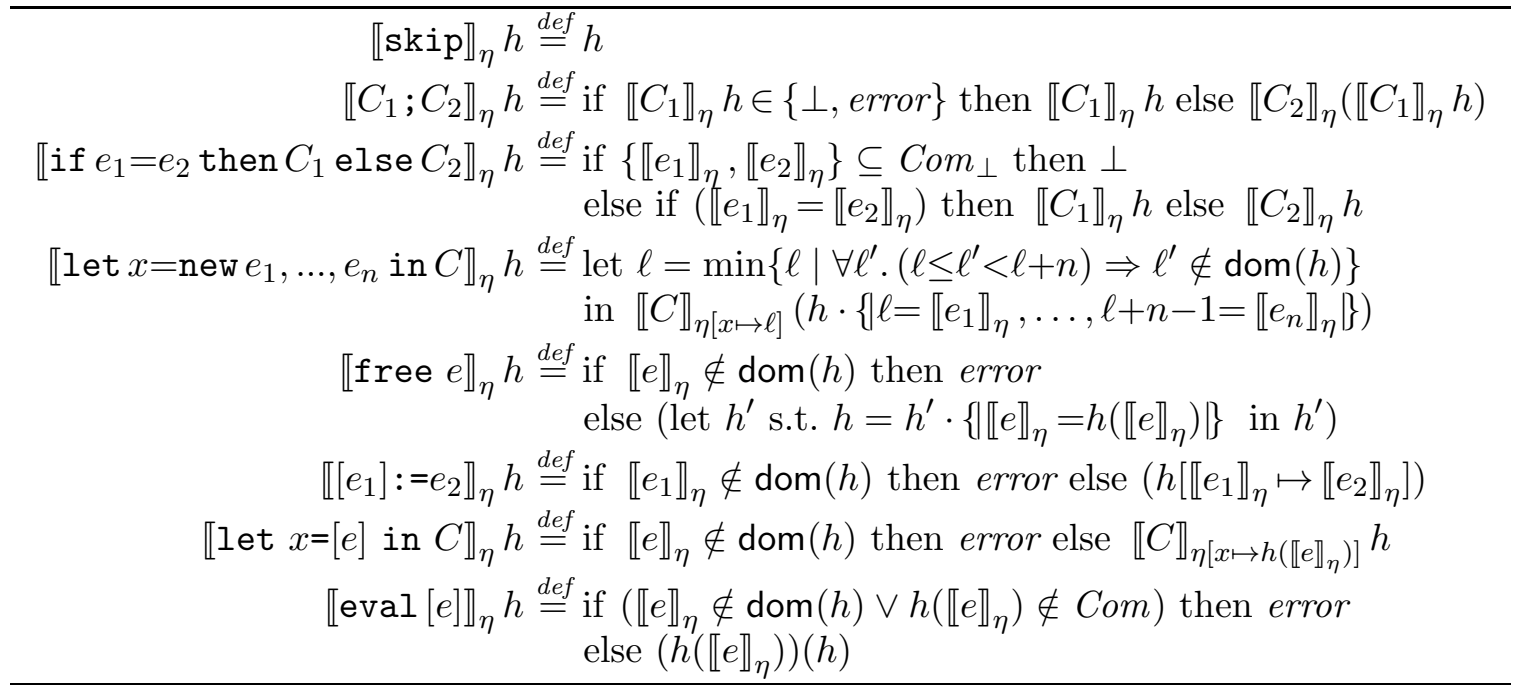

FIGURE 7. Interpretation of commands $\llbracket C \rrbracket_{\eta} \in$ Heap $\multimap T_{\text {err }}($ Heap $)$

and is the same as in our previous work [5]. That is, commands denote strict continuous functions $\llbracket C \rrbracket_{\eta} \in$ Heap $\multimap T_{\text {err }}($ Heap $)$ where

$$
\text { Heap }=\operatorname{Rec}(\mathrm{Val}) \quad \mathrm{Val}=\text { Integer }_{\perp} \oplus \mathrm{Com}_{\perp} \quad \mathrm{Com}=\text { Heap } \multimap T_{\text {err }}(\text { Heap })
$$

In these equations, $T_{e r r}(D)=D \oplus\{\text { error }\}_{\perp}$ denotes the error monad, and $\operatorname{Rec}(D)$ denotes records with entries from $D$ and labelled by positive natural numbers. Formally, $\operatorname{Rec}(D)=$ $\left(\sum_{N \subseteq_{\text {fin } N a t s}+}\left(N \rightarrow D_{\downarrow}\right)\right)_{\perp}$ where $\left(N \rightarrow D_{\downarrow}\right)$ is the cpo of maps from the finite address set $N$ to the cpo $D_{\downarrow}=D-\{\perp\}$ of non-bottom elements of $D$. We use some evident record notation, such as $\left\{\left|\ell_{1}=d_{1}, \ldots, \ell_{n}=d_{n}\right|\right\}$ for the record mapping label $\ell_{i}$ to $d_{i}$, and $\operatorname{dom}(r)$ for the set of labels of a record $r$. The disjointness predicate $r \# r^{\prime}$ on records holds if $r$ and $r^{\prime}$ are not $\perp$ and have disjoint domains, and a partial combining operation $r \cdot r^{\prime}$ is defined by

$$
r \cdot r^{\prime} \stackrel{\text { def }}{=} \text { if } r \# r^{\prime} \text { then } r \cup r^{\prime} \text { else } \perp \text {. }
$$

The interpretation of commands is repeated in Figure 7 (assuming $h \neq \perp$ ) and below we point out where this interpretation deviates from the norm. Firstly, the new statement uses a deterministic allocator which, however, can not be controlled by the programmen 8 which is important to ensure that allocation respects the frame rule. Any deterministic allocator would work here, but note that in our denotational semantics we can only work with deterministic allocation. The semantics of the if statement is divergence if one of the expressions in the test is a command. If we wanted to raise an error in this case (which is more appropriate), we would have to include type checking into the logic due to our fault avoiding semantics of triples. We decided not do this here as it would clutter the rules with type checking assertions like int $(e)$ or $\operatorname{com}(e)$ which are true in case expression $e$ is an integer valued expression or a command, respectively.

\footnotetext{
${ }^{8}$ This means that there is no way to stipulate what the new location is as this must depend solely on the already allocated locations.
} 
The interpretation of expressions is entirely standard with the exception of the quote operation, ' $C$ ', that uses the injection of Com into Val. Thus, the semantic equations for expressions are omitted.

A solution to equation (4.1) for Heap can be obtained by the usual inverse limit construction [29] in the category $\mathbf{C p p o}_{\perp}$. This solution is an SFP domain (e.g., [31]), and thus comes equipped with an increasing chain $\pi_{n}$ : Heap $\rightarrow$ Heap of continuous projection maps, satisfying $\pi_{0}=\perp, \bigsqcup_{n \in \omega} \pi_{n}=i d_{\text {Heap }}$, and $\pi_{n} \circ \pi_{m}=\pi_{\min \{n, m\}}$. The image of each $\pi_{n}$ is finite, hence each $\pi_{n}(h)$ is a compact element of Heap. Moreover, the projections are compatible with composition of heaps: we have $\pi_{n}\left(h \cdot h^{\prime}\right)=\pi_{n}(h) \cdot \pi_{n}\left(h^{\prime}\right)$ for all $h, h^{\prime}$.

4.3. Semantic domain for assertions. A subset $p \subseteq$ Heap is admissible if $\perp \in p$ and if $p$ is closed under taking least upper bounds of $\omega$-chains. It is uniform [6] if it is closed under the projections, i.e., if $h \in p$ implies $\pi_{n}(h) \in p$ for all $n$. We write $U A d m$ for the set of all uniform admissible subsets of Heap. For $p \in U A d m, p_{[n]}$ denotes the image of $p$ under $\pi_{n}$. Note that uniformity means $p_{[n]} \subseteq p$, and that $p_{[n]} \in U A d m$. We may regard any subset $p \subseteq$ Heap (not necessarily uniform or admissible) as a subset of $T_{\text {err }}($ Heap ) in the evident way.

The uniform admissible subsets will form the basic building block when interpreting the assertions of our logic. As we have already described informally above, assertions in general depend on invariants for stored code. Thus, the space of semantic predicates Pred will consist of functions $W \rightarrow U A d m$ from a set of "worlds," describing the invariants, to the collection of uniform admissible subsets of heaps. But, the invariants for stored code are themselves semantic predicates, and the interaction between Pred and $W$ is governed by (the semantics of) $\otimes$. Hence we seek a space of worlds $W$ that is "the same" as $W \rightarrow U A d m$. We obtain such a $W$ using metric spaces.

Recall that a 1-bounded ultrametric space $(X, d)$ is a metric space where the distance function $d: X \times X \rightarrow \mathbb{R}$ takes values in the closed interval $[0,1]$ and satisfies the strong triangle inequality $d(x, y) \leq \max \{d(x, z), d(z, y)\}$, for all $x, y, z \in X$. An (ultra-) metric space is complete if every Cauchy sequence has a limit. A function $f: X_{1} \rightarrow X_{2}$ between metric spaces $\left(X_{1}, d_{1}\right)$ and $\left(X_{2}, d_{2}\right)$ is non-expansive if for all $x, y \in X_{1}, d_{2}(f(x), f(y)) \leq$ $d_{1}(x, y)$. It is contractive if for some $\delta<1, d_{2}(f(x), f(y)) \leq \delta \cdot d_{1}(x, y)$ for all $x, y \in X_{1}$. By the Banach fixed point theorem, every contractive function $f: X \rightarrow X$ on a non-empty and complete metric space $(X, d)$ has a unique fixed point.

The complete, 1-bounded, non-empty ultrametric spaces and non-expansive functions between them form a Cartesian closed category CBUlt. Products in CBUlt are given by the set-theoretic product where the distance is the maximum of the componentwise distances. The exponentials are given by the non-expansive functions equipped with the sup-metric, i.e., the exponential $\left(X_{1}, d_{1}\right) \rightarrow\left(X_{2}, d_{2}\right)$ has the set of non-expansive functions from $\left(X_{1}, d_{1}\right)$ to $\left(X_{2}, d_{2}\right)$ as underlying set, and distance function: $d_{X_{1} \rightarrow X_{2}}(f, g)=$ $\sup \left\{d_{2}(f(x), g(x)) \mid x \in X_{1}\right\}$. A functor $F: C B U l t^{o p} \times C B U l t \rightarrow$ CBUlt is locally nonexpansive if $d\left(F(f, g), F\left(f^{\prime}, g^{\prime}\right)\right) \leq \max \left\{d\left(f, f^{\prime}\right), d\left(g, g^{\prime}\right)\right\}$ for all non-expansive $f, f^{\prime}, g, g^{\prime}$, and it is locally contractive if $d\left(F(f, g), F\left(f^{\prime}, g^{\prime}\right)\right) \leq \delta \cdot \max \left\{d\left(f, f^{\prime}\right), d\left(g, g^{\prime}\right)\right\}$ for some $\delta<1$. The functor that results from composing a locally non-expansive functor with a locally contractive one is locally contractive. By multiplication of the distance function of an ultrametric space $(X, d)$ with a shrinking factor $\delta<1$ one obtains a new ultrametric 
space, $\delta \cdot(X, d)=\left(X, d^{\prime}\right)$ where $d^{\prime}(x, y)=\delta \cdot d(x, y)$. Using this operation, a locally contractive functor $(\delta \cdot F)\left(X_{1}, X_{2}\right)=\delta \cdot\left(F\left(X_{1}, X_{2}\right)\right)$ can be obtained from any locally non-expansive functor $F$.

The set UAdm of uniform admissible subsets of Heap becomes a complete, 1-bounded ultrametric space when equipped with the following distance function:

$$
d(p, q)= \begin{cases}2^{-\max \left\{i \in \omega \mid p_{[i]}=q_{[i]}\right\}} & \text { if } p \neq q \\ 0 & \text { otherwise }\end{cases}
$$

Note that $d$ is well-defined: first, because $\pi_{0}=\perp$ and $\perp \in p$ for all $p \in U A d m$ the set $\left\{i \in \omega \mid p_{[i]}=q_{[i]}\right\}$ is non-empty; second, this set is finite, because $p \neq q$ implies $p_{[i]} \neq q_{[i]}$ for all sufficiently large $i$ by the uniformity of $p, q$ and the fact that the limit of the projections $\pi_{i}$ is the identity on Heap.

Theorem 4.1 (Existence of recursive worlds). There exists an ultrametric space $W$ and an isomorphism $\iota$ from $\frac{1}{2} \cdot(W \rightarrow U A d m)$ to $W$ in CBUlt.

Proof. By an application of America \& Rutten's existence theorem for fixed points of locally contractive functors [1], applied to the functor $F(X, Y)=\frac{1}{2} \cdot(X \rightarrow U A d m)$ on CBUlt. See [6] for details of a similar recent application.

We write Pred for $\frac{1}{2} \cdot(W \rightarrow U A d m)$ and $\iota^{-1}: W \cong$ Pred for the inverse to $\iota$.

Definition 4.2 (Approximate equality, [6]). For an ultrametric space $(X, d)$ and $n \in \omega$ we use the notation $x \stackrel{n}{=} y$ to mean that $d(x, y) \leq 2^{-n}$.

We conclude this subsection with a number of simple but useful observations, which will be used repeatedly in the following proofs. By the ultrametric inequality, each $\stackrel{n}{=}$ is an equivalence relation on $X$. Moreover, if $n \leq m$ then $\stackrel{n}{=} \supseteq \stackrel{m}{=}$, and $x=y$ if and only if $x \stackrel{n}{=} y$ for all $n \in \omega$. Since all non-zero distances in $U A d m$ are of the form $2^{-n}$ for some $n \in \omega$, this is also the case for the distance function on $W$. Therefore, to show that a map is non-expansive it suffices to show that $f(x) \stackrel{n}{=} f(y)$ whenever $x \stackrel{n}{=} y$. Finally, the definition of Pred has the following consequence: for $p, q \in$ Pred, $p \stackrel{n}{=} q$ holds if and only if $p(w) \stackrel{n-1}{=} q(w)$ for all $w \in W$.

4.4. Separating conjunction and invariant extension. For $p, q \in U A d m$, the separating conjunction $p * q$ is defined as usual, by

$$
h \in p * q \stackrel{\text { def }}{\Leftrightarrow} \exists h_{1}, h_{2} . h=h_{1} \cdot h_{2} \wedge h_{1} \in p \wedge h_{2} \in q .
$$

This operation is lifted to non-expansive functions $p_{1}, p_{2} \in$ Pred pointwise, by letting $\left(p_{1} * p_{2}\right)(w)=p_{1}(w) * p_{2}(w)$. This lifting is well-defined, and moreover determines a nonexpansive operation on the space Pred:

Lemma 4.3 (Separating conjunction). If $p, q \in$ Pred then $p * q \in$ Pred. Moreover, the assignment of $p, q$ to $p * q$ is a non-expansive operation on Pred.

Proof. As a preliminary step one shows that separating conjunction on UAdm is welldefined, i.e., if $p, q \in U A d m$ then so is $p * q$ : The admissibility of $p * q$ follows from $\perp=\perp \cdot \perp$, and from the fact that (non- $\perp$ ) heaps are only comparable with respect to the order on Heap if they have equal (finite) domains. More precisely, any chain $h_{0} \sqsubseteq h_{1} \sqsubseteq \ldots$ in $p * q$ must have a subsequence $\left(h_{i_{k}}\right)_{k}=\left(h_{i_{k}}^{\prime} \cdot h_{i_{k}}^{\prime \prime}\right)_{k}$ that splits into chains $h_{i_{1}}^{\prime} \sqsubseteq h_{i_{2}}^{\prime} \sqsubseteq \ldots$ in $p$ and 
$h_{i_{1}}^{\prime \prime} \sqsubseteq h_{i_{2}}^{\prime \prime} \sqsubseteq \ldots$ in $q$. The combination of their respective lubs in $p$ and $q$ is the lub of the $h_{k}$ 's, and therefore in $p * q$ by the admissibility of $p$ and $q$. The uniformity of $p * q$ is a consequence of the equation $\pi_{n}\left(h_{1} \cdot h_{2}\right)=\pi_{n}\left(h_{1}\right) \cdot \pi_{n}\left(h_{2}\right) \in p * q$.

We now show that for $p, q \in \operatorname{Pred}, p * q$ is a non-expansive function. Suppose $w, w^{\prime} \in W$ such that $w \stackrel{n}{=} w^{\prime}$, and suppose $\pi_{n}(h) \in(p * q)(w)=p(w) * q(w)$. We must show that $\pi_{n}(h) \in(p * q)\left(w^{\prime}\right)$. By definition of $*$ on $U A d m$ there exist $h_{1} \in p(w)$ and $h_{2} \in q(w)$ such that $\pi_{n}(h)=h_{1} \cdot h_{2}$. By uniformity, we also have $\pi_{n}\left(h_{1}\right) \in p(w)$ and $\pi_{n}\left(h_{2}\right) \in q(w)$. Since we assumed $w \stackrel{n}{=} w^{\prime}$, this yields

$$
\pi_{n}\left(h_{1} \cdot h_{2}\right)=\pi_{n}\left(h_{1}\right) \cdot \pi_{n}\left(h_{2}\right) \in p\left(w^{\prime}\right) * q\left(w^{\prime}\right)=(p * q)\left(w^{\prime}\right) .
$$

Finally, since $\pi_{n}(h)=\pi_{n}\left(\pi_{n}(h)\right)=\pi_{n}\left(h_{1} \cdot h_{2}\right)$, the statement $\pi_{n}(h) \in(p * q)\left(w^{\prime}\right)$ follows.

To see that separating conjunction is non-expansive, assume that $p \stackrel{n}{=} p^{\prime}$ and $q \stackrel{n}{=} q^{\prime}$ for arbitrary $p, p^{\prime}, q, q^{\prime} \in$ Pred. We must show that $p * q \stackrel{n}{=} p^{\prime} * q^{\prime}$. Since Pred $=\frac{1}{2} \cdot(W \rightarrow U A d m)$ we can equivalently show that $p(w) * q(w) \stackrel{n=1}{=} p^{\prime}(w) * q^{\prime}(w)$ for all $w \in W$. This follows from the assumption that $p \stackrel{n}{=} p^{\prime}$ and $q \stackrel{n}{=} q^{\prime}$ and the fact that $\pi_{n-1}(h)=\pi_{n-1}\left(h_{1}\right) \cdot \pi_{n-1}\left(h_{2}\right)$ whenever $h=h_{1} \cdot h_{2}$.

The corresponding unit for the lifted separating conjunction is the non-expansive function $e m p=\lambda w .\{\{||\}, \perp\}$, i.e., $p * e m p=e m p * p=p$ holds for all $p \in$ Pred. We let the world $e m p \stackrel{\text { def }}{=} \iota(e m p)$ be its image under the isomorphism.

The following lemma introduces semantic analogues of the syntactic invariant extension operation $P \otimes R$ and the invariant combination $R \circ R^{\prime}$.

Lemma 4.4 (Invariant combination and invariant extension). There exists a non-expansive map $\circ: W \times W \rightarrow W$ and a map $\otimes:$ Pred $\times W \rightarrow$ Pred that is non-expansive in its first and contractive in its second argument, satisfying the equations

$$
r \circ r^{\prime}=\iota\left(\iota^{-1}(r) \otimes r^{\prime} * \iota^{-1}\left(r^{\prime}\right)\right) \quad \text { and } \quad(p \otimes r)(w)=p(r \circ w)
$$

for all $p \in$ Pred and $r, r^{\prime} \in W$.

Proof. The defining equations of both operations give rise to contractive maps, which have (unique) fixed points by Banach's fixed point theorem. More precisely, consider the endofunction ${ }^{-}$on the function space $W \times W \rightarrow W$, defined for all $\circ \in(W \times W \rightarrow W)$ and all $r, r^{\prime} \in W$ by

$$
r \bar{\circ} r^{\prime}=\iota\left(\left(\lambda w \cdot \iota^{-1}(r)\left(r^{\prime} \circ w\right)\right) * \iota^{-1}\left(r^{\prime}\right)\right) .
$$

Note that $\bar{\sigma}$ is indeed a non-expansive function, i.e., an element of the function space $(W \times W \rightarrow W)$ : if $r \stackrel{n}{=} s$ and $r^{\prime} \stackrel{n}{=} s^{\prime}$ then $r^{\prime} \circ w \stackrel{n}{=} s^{\prime} \circ w$ holds in $W$, for all $w \in W$, and $\iota^{-1}(r) \stackrel{n}{=} \iota^{-1}(s)$ and $\iota^{-1}\left(r^{\prime}\right) \stackrel{n}{=} \iota^{-1}\left(s^{\prime}\right)$ holds in Pred. Since separating conjunction is non-expansive by Lemma 4.3, the approximate equality

$$
\left(\lambda w \cdot \iota^{-1}(r)\left(r^{\prime} \circ w\right)\right) * \iota^{-1}\left(r^{\prime}\right) \stackrel{n-1}{=}\left(\lambda w \cdot \iota^{-1}(s)\left(s^{\prime} \circ w\right)\right) * \iota^{-1}\left(s^{\prime}\right)
$$

holds in $W \rightarrow U A d m$, so that $r \bar{\circ} r^{\prime} \stackrel{n}{=} s \bar{\circ} s^{\prime}$ in $W$.

We show that the function $:$ is contractive. Assume that $\circ_{1} \stackrel{n}{=} \circ_{2}$ holds in $W \times W \rightarrow W$; we must show that $\overline{o_{1}} \stackrel{n+1}{=} \overline{o_{2}}$. Let $r, r^{\prime} \in W$ be arbitrary. Then by the sup-metric on $W \times W \rightarrow W$ it suffices to prove that $r \overline{\mathrm{o}_{1}} r^{\prime} \stackrel{n+1}{=} r \overline{\mathrm{o}_{2}} r^{\prime}$ holds in $W$, or equivalently, that

$$
\left(\lambda w \cdot \iota^{-1}(r)\left(r^{\prime} \circ_{1} w\right)\right) * \iota^{-1}\left(r^{\prime}\right) \stackrel{n}{=}\left(\lambda w \cdot \iota^{-1}(r)\left(r^{\prime} \circ_{2} w\right)\right) * \iota^{-1}\left(r^{\prime}\right)
$$


holds in $W \rightarrow U A d m$. By the non-expansiveness of separating conjunction (Lemma 4.3) and the sup-metric on $W \rightarrow U A d m$, this follows since $r^{\prime} \circ_{1} w \stackrel{n}{=} r^{\prime} \circ_{2} w$ holds for all $w \in W$ by the assumption that $\circ_{1} \stackrel{n}{=} \circ_{2}$, and hence $\iota^{-1}(r)\left(r^{\prime} \circ_{1} w\right) \stackrel{n}{=} \iota^{-1}(r)\left(r^{\prime} \circ_{2} w\right)$ holds.

By contractiveness of ${ }^{-}$and the Banach fixed point theorem, there exists a unique nonexpansive map o satisfying $r \circ r^{\prime}=r \bar{\circ} r^{\prime}$. We can now define the operation $\otimes:$ Pred $\times W \rightarrow$ Pred by $p \otimes r \stackrel{\text { def }}{=} \lambda w . p(r \circ w)$ for all $p \in$ Pred and $r \in W$, from which the required equivalences follow:

$$
r \circ r^{\prime}=r \bar{\circ} r^{\prime}=\iota\left(\lambda w \cdot \iota^{-1}(r)\left(r^{\prime} \circ w\right) * \iota^{-1}\left(r^{\prime}\right)\right)=\iota\left(\iota^{-1}(r) \otimes r^{\prime} * \iota^{-1}\left(r^{\prime}\right)\right)
$$

Finally, we note that if $p \stackrel{n}{=} p^{\prime}$ and $r \stackrel{m}{=} r^{\prime}$ then $p \otimes r \stackrel{k}{=} p^{\prime} \otimes r^{\prime}$ for $k=\min \{n, m+1\}$, i.e., the operation is non-expansive in its first argument and contractive in its second argument. To see this, suppose $p \stackrel{n}{=} p^{\prime}$ holds in Pred and $r \stackrel{m}{=} r^{\prime}$ holds in $W$. Without loss of generality we may assume $n>0$, so that $p \stackrel{n-1}{=} p^{\prime}$ holds in $W \rightarrow U A d m$. By non-expansiveness of $\circ$ it follows that $r \circ w \stackrel{m}{=} r^{\prime} \circ w$ for all $w$, and therefore $\lambda w \cdot p(r \circ w) \stackrel{\min \{n-1, m\}}{=} \lambda w \cdot p^{\prime}\left(r^{\prime} \circ w\right)$ in $W \rightarrow U A d m$. Hence $p \otimes r \stackrel{\min \{n, m+1\}}{=} p^{\prime} \otimes r^{\prime}$ holds in Pred as required.

The following lemma establishes key properties of the two operations $\circ$ and $\otimes$ that we defined in Lemma 4.4. These properties provide a semantic explanation of the distribution axioms given in Figure 2 ,

Lemma 4.5 (Monoid structure and monoid action). ( $W, \circ, e m p)$ is a monoid in CBUlt. Moreover, $\otimes$ is an action of this monoid on Pred.

Proof. First, emp is a left-unit for $\circ$, since

$$
e m p \circ r=\iota\left(\left(\lambda w \cdot \iota^{-1}(e m p)(r \circ w)\right) * \iota^{-1}(r)\right)=\iota\left(\iota^{-1}(r)\right)=r .
$$

Using this fact, it is easy to prove that it is also a right-unit for the o operation:

$$
r \circ e m p=\iota\left(\lambda w \cdot \iota^{-1}(r)(e m p \circ w) * \iota^{-1}(e m p)\right)=\iota\left(\lambda w \cdot \iota^{-1}(r)(w) * e m p\right)=r .
$$

Next, we prove by induction that for all $n \in \omega$, ० is associative up to distance $2^{-n}$, from which associativity follows. By the 1-boundedness of $W$ the base case is clear. For the inductive step $n>0$, by definition of the distance function on Pred it suffices to show that for all $w \in W, \iota^{-1}((r \circ s) \circ t)(w) \stackrel{n-1}{=} \iota^{-1}(r \circ(s \circ t))(w)$. This equation follows from the definition of $\circ$ as follows:

$$
\begin{aligned}
\iota^{-1}((r \circ s) \circ t)(w) & =\iota^{-1}(r \circ s)(t \circ w) * \iota^{-1}(t)(w) \\
& =\iota^{-1}(r)(s \circ(t \circ w)) * \iota^{-1}(s)(t \circ w) * \iota^{-1}(t)(w) \\
& =\iota^{-1}(r)(s \circ(t \circ w)) * \iota^{-1}(s \circ t)(w) \\
& \stackrel{n-1}{=} \iota^{-1}(r)((s \circ t) \circ w) * \iota^{-1}(s \circ t)(w) \\
& =\iota^{-1}(r \circ(s \circ t))(w) .
\end{aligned}
$$

The second last step in this derivation is by the inductive hypothesis, using the nonexpansiveness of $\iota^{-1}(r)$.

That $\otimes$ forms an action of $W$ on Pred follows from these properties of $\circ$. First, $p \otimes e m p=$ $\lambda w \cdot p(e m p \circ w)=p$ since $e m p$ is a unit for $\circ$. Second,

$$
(p \otimes r) \otimes s=\lambda w \cdot p(r \circ(s \circ w))=\lambda w \cdot p((r \circ s) \circ w)=p \otimes(r \circ s)
$$


by the associativity of $\circ$.

4.5. Semantics of triples and assertions. Since assertions appear in the pre- and postconditions of Hoare triples, and triples can be nested inside assertions, the interpretation of assertions and the validity of triples must be defined simultaneously. To achieve this, we first define a notion of fault-avoiding semantic triple.

Definition 4.6 (Semantic triple). A semantic Hoare triple consists of predicates $p, q \in$ Pred and a strict continuous function $c \in$ Heap $\multimap T_{\text {err }}($ Heap), written $\{p\} c\{q\}$. For $w \in W$, a semantic triple $\{p\} c\{q\}$ is forced by $w$, written $w \models\{p\} c\{q\}$, if for all $r \in U A d m$ and all $h \in$ Heap:

$$
h \in p(w) * \iota^{-1}(w)(e m p) * r \Rightarrow c(h) \in \operatorname{Ad}\left(q(w) * \iota^{-1}(w)(e m p) * r\right),
$$

where $\operatorname{Ad}(r)$ denotes the least downward closed and admissible set of heaps containing $r$. A semantic triple is valid, written $\models\{p\} c\{q\}$, if $w \models\{p\} c\{q\}$ for all $w \in W$. We extend semantic triples from Com $=$ Heap $\multimap T_{\text {err }}$ (Heap) to all $d \in V a l$, by $w \models\{p\} d\{q\}$ iff $d=c$ for some command $c \in C o m$ and $w \models\{p\} c\{q\}$.

A triple holds approximately up to level $k, w \models_{k}\{p\} d\{q\}$, if $w \models\{p\} \pi_{k} ; d ; \pi_{k}\{q\}$.

Thus, semantic triples bake in the first-order frame property (by conjoining $r$ ), and "close" the "open" recursion (by applying the world $w$, on which the triple implicitly depends, to emp). The semantics also ensures that if a triple holds the command in question must not have produced error as result. One calls such a semantics fault-avoiding and this is one of the intrinsic features of Separation Logic. In our case fault-avoidance follows directly from the fact that semantics of assertions indexed by worlds lives in $U A d m$ that ranges over heaps and does not include value error. The admissible downward closure that is applied to the entire post-condition is in line with a partial correctness interpretation of triples. In particular, it entails that the sets $\left\{c \in \operatorname{Com} \mid w \models_{k}\{p\} c\{q\}\right\}$ and $\{c \in \operatorname{Com} \mid w \models\{p\} c\{q\}\}$ are admissible and downward closed subsets of Com.

Since there is a closure operation applied to the post-condition of semantic triples, but no similar closure used in the pre-condition, it may not be immediate that proved commands compose. The following characterisation is helpful, for instance when proving soundness of the rule of sequential composition.

Lemma 4.7 (Closure). If $f: D \multimap D^{\prime}$ is a strict continuous function, $q \subseteq D^{\prime}$ is an admissible and downwards closed subset of $D^{\prime}$, and $p \subseteq D$ is an arbitrary subset of $D$, then $f(p) \subseteq q$ implies $f(\operatorname{Ad}(p)) \subseteq q$.

Proof. Since $f$ is continuous, the pre-image $f^{-1}(q)$ of $q$ is admissible and downward closed. From the assumption that $f(p) \subseteq q$ it follows that $p \subseteq f^{-1}(q)$, and thus $\operatorname{Ad}(p) \subseteq f^{-1}(q)$ as the former is by definition the least admissible and downward closed subset of $D$ containing $p$. Thus, if $h \in \operatorname{Ad}(p)$ then $f(h) \in q$.

Observe that $w \models_{k}\{p\} d\{q\}$ provides indeed an approximation of the judgement $w \models$ $\{p\} c\{q\}$, in the sense that $w \models\{p\} c\{q\}$ is equivalent to $\forall k \in \omega . w \models_{k}\{p\} c\{q\}$. Finally, semantic triples are non-expansive, in the sense that if $w^{n+1}=w^{\prime}$ and $w \models_{n}\{p\} c\{q\}$, then $w^{\prime} \models_{n}\{p\} c\{q\}$; they are similarly non-expansive in the pre- and post-conditions $p$ and $q$. This observation plays a key role in the following definition of the semantics of nested triples. 
Lemma 4.8 (Non-expansiveness of semantic triples). Let $w, w^{\prime} \in W$ such that $w^{n+1}=w^{\prime}$. Let $p, p^{\prime}, q, q^{\prime} \in$ Pred be such that $p \stackrel{n}{=} p^{\prime}$ and $q \stackrel{n}{=} q^{\prime}$. If $w=_{n}\{p\} c\{q\}$, then $w^{\prime} \models_{n}\left\{p^{\prime}\right\} c\left\{q^{\prime}\right\}$.

Proof. Let $w, w^{\prime}, p, p^{\prime}, q$ and $q^{\prime}$ be as in the statement of the lemma, and let $c:$ Heap $T_{\text {err }}($ Heap $)$ be such that $w \models_{n}\{p\} c\{q\}$. To prove that $w^{\prime} \models_{n}\{p\} c^{\prime}\{q\}$, suppose $r \in$ $U A d m$ and $h \in$ Heap are such that $h \in p^{\prime}\left(w^{\prime}\right) * \iota^{-1}\left(w^{\prime}\right)(e m p) * r$. We have to show that $\pi_{n}\left(c\left(\pi_{n} h\right)\right) \in \operatorname{Ad}\left(q^{\prime}\left(w^{\prime}\right) * \iota^{-1}\left(w^{\prime}\right)(e m p) * r\right)$.

Since $w^{n+1}=w^{\prime}$ holds by assumption, we have $\iota^{-1}\left(w^{\prime}\right)(e m p) \stackrel{n}{=} \iota^{-1}(w)(e m p)$. Hence, by the non-expansiveness of $p$, by the assumption $p \stackrel{n}{=} p^{\prime}$, and by the compatibility of the heap combination operation with projections, we have $\pi_{n}(h) \in p(w) * \iota^{-1}(w)(e m p) * r$. By the assumption that $w \models_{n}\{p\} c\{q\}$ and since $\pi_{n} \circ \pi_{n}=\pi_{n}$, this yields $\pi_{n}\left(c\left(\pi_{n} h\right)\right) \in$ $\operatorname{Ad}\left(q(w) * \iota^{-1}(w)(e m p) * r\right)$. Using the non-expansiveness of $q$, the assumption $q \stackrel{n}{=} q^{\prime}$, uniformity of $r$, and the fact that $\iota^{-1}(w)(e m p) \stackrel{n}{=} \iota^{-1}\left(w^{\prime}\right)(e m p)$, we know that $\pi_{n}\left(h^{\prime}\right) \in$ $q^{\prime}\left(w^{\prime}\right) * \iota^{-1}\left(w^{\prime}\right)(e m p) * r$ holds whenever $h^{\prime} \in q(w) * \iota^{-1}(w)(e m p) * r$. Thus, using $\pi_{n} \circ \pi_{n}=\pi_{n}$ again, $\pi_{n}\left(c\left(\pi_{n}(h)\right)\right) \in \operatorname{Ad}\left(q^{\prime}\left(w^{\prime}\right) * \iota^{-1}\left(w^{\prime}\right)(e m p) * r\right)$ holds by Lemma 4.7 and the continuity of the projection $\pi_{n}$.

Assertions (without free relation variables) are interpreted as elements $\llbracket P \rrbracket_{\eta} \in$ Pred. More generally, assume that the free relation variables of $P$ are contained in $\Xi=X_{1}, \ldots, X_{n}$, where the arity of $X_{i}$ is $n_{i}$. Then $P$ denotes a non-expansive function from $\prod_{X_{i} \in \Xi} \operatorname{Pred}^{\left(\operatorname{Val}^{\left.n_{i}\right)}\right.}$ to Pred. Note that $(U A d m, \subseteq$ ) is a complete Heyting algebra (as shown in Appendix B.1, Lemma B.1). Using the pointwise extension of the operations of this algebra to the set of non-expansive functions $W \rightarrow U A d m$, we also obtain a complete Heyting algebra on Pred $=\frac{1}{2} \cdot(W \rightarrow U A d m)$ which soundly models the intuitionistic predicate part of the assertion logic. (See Appendix B.1, Lemma B.2 for details.) The monoid action of $W$ on Pred serves to model the invariant extension of the assertion logic.

Remark 4.9. While UAdm (and hence Pred) is a complete Heyting algebra, it is not a complete Heyting BI algebra, as usually assumed for the interpretation of the assertion language in separation logic [22]. More precisely, what is missing is the right adjoint ("magic wand") for the monoid operation $*$ : the candidate operation,

$$
p * *=\left\{h \mid \forall n \in \omega . \forall h^{\prime} \in \text { Heap. if } \pi_{n}\left(h^{\prime}\right) \in p \wedge \pi_{n}(h) \# \pi_{n}\left(h^{\prime}\right) \text { then } \pi_{n}\left(h \cdot h^{\prime}\right) \in q\right\},
$$

alas, fails to be non-expansive. This is a particularly annoying shortcoming of our model since this spatial implication is important when dealing with shared memory. For instance, $(P * Q) *(P * R) * P$ expresses that $R$ and $Q$ overlap in shared part $P$. Recently, we have constructed an alternative model of our logic, based on an operational semantics of the programming language and using the ideas of step-indexing, where the right adjoint does exist.

In order to define an interpretation of nested triples we use the following definition:

Definition 4.10 (Rank of a heap). If $h$ is a compact element of Heap, then the least $n$ for which $\pi_{n}(h)=h$ is the rank of $h$, abbreviated $r n k(h)$, otherwise the rank is undefined.

The interpretation of assertions is spelled out in detail in Figure 8 . The interpretation of a nested triple $\{P\} e\{Q\}$ is not independent of the heap, unlike the (more traditional) semantics of "top-level" triples, i.e. $\models\{p\} c\{q\}$. More precisely, the definition in Figure 8 means that triples as assertions depend on the rank of the current heap. This is necessary to provide a non-expansive function from $W$ to $U A d m$. Simpler definitions of the interpretation 


$$
\begin{aligned}
& \llbracket X(\vec{e}) \rrbracket_{\eta, \rho} w=\rho(X)\left(\llbracket \vec{e} \rrbracket_{\eta}\right) w \\
& \llbracket \text { false } \rrbracket_{\eta, \rho} w=\{\perp\} \\
& \llbracket \text { true } \rrbracket_{\eta, \rho} w=\text { Heap } \\
& \llbracket P \vee Q \rrbracket_{\eta, \rho} w=\llbracket P \rrbracket_{\eta, \rho} w \cup \llbracket Q \rrbracket_{\eta, \rho} w \\
& \llbracket P \wedge Q \rrbracket_{\eta, \rho} w=\llbracket P \rrbracket_{\eta, \rho} w \cap \llbracket Q \rrbracket_{\eta, \rho} w \\
& \llbracket P \Rightarrow Q \rrbracket_{\eta, \rho} w=\left\{h \mid \forall n \in \omega . \pi_{n}(h) \in \llbracket P \rrbracket_{\eta, \rho} w \text { implies } \pi_{n}(h) \in \llbracket Q \rrbracket_{\eta, \rho} w\right\} \\
& \llbracket \forall x . P \rrbracket_{\eta, \rho} w=\bigcap_{d \in \text { Val }} \llbracket P \rrbracket_{\eta[x:=d], \rho} w \\
& \llbracket \exists x . P \rrbracket_{\eta, \rho} w=\left\{h \mid \forall n \in \omega . \pi_{n}(h) \in \bigcup_{d \in \text { Val }} \llbracket P \rrbracket_{\eta[x:=d], \rho} w\right\} \\
& \llbracket e_{1}=e_{2} \rrbracket_{\eta, \rho} w=\left\{h \mid h \neq \perp \Rightarrow \llbracket e_{1} \rrbracket_{\eta}=\llbracket e_{2} \rrbracket_{\eta}\right\} \\
& \llbracket e_{1} \mapsto e_{2} \rrbracket_{\eta, \rho} w=\left\{h \mid h \sqsubseteq\left\{\left|\llbracket e_{1} \rrbracket_{\eta}=\llbracket e_{2} \rrbracket_{\eta}\right|\right\}\right\} \\
& \llbracket e m p \rrbracket_{\eta, \rho} w=\{\{||\}, \perp\} \\
& \llbracket P * Q \rrbracket_{\eta, \rho} w=\llbracket P \rrbracket_{\eta, \rho} w * \llbracket Q \rrbracket_{\eta, \rho} w \\
& \llbracket\{P\} e\{Q\} \rrbracket_{\eta, \rho} w=\operatorname{Ad}\left\{h \in \operatorname{Heap} \mid \operatorname{rnk}(h)>0 \Rightarrow w \models_{r n k(h)-1}\left\{\llbracket P \rrbracket_{\eta, \rho}\right\} \llbracket e \rrbracket_{\eta}\left\{\llbracket Q \rrbracket_{\eta, \rho}\right\}\right\} \\
& \llbracket P \otimes Q \rrbracket_{\eta, \rho} w=\left(\llbracket P \rrbracket_{\eta, \rho} \otimes \iota\left(\llbracket Q \rrbracket_{\eta, \rho}\right)\right) w \\
& \llbracket(\mu X(\vec{x}) \cdot P)(\vec{e}) \rrbracket_{\eta, \rho} w=f i x\left(\lambda q, \vec{d} \cdot \llbracket P \rrbracket_{\eta[\vec{x}:=\vec{d}], \rho[X:=q]}\right)\left(\llbracket \vec{e} \rrbracket_{\eta}\right) w
\end{aligned}
$$

FiguRE 8. Semantics of assertions

of triples, like $\left\{h \in\right.$ Heap $\left.\mid w \models\left\{\llbracket P \rrbracket_{\eta, \rho}\right\} \llbracket e \rrbracket_{\eta}\left\{\llbracket Q \rrbracket_{\eta, \rho}\right\}\right\}$, are heap independent but not non-expansive. A similar approach has been taken in [6] to force non-expansiveness for a reference type constructor for ML-style references. We discuss the ramifications of this choice in Section 5. Note also that the only atomic assertions that depend on the world $w$ are triples, as they are the only ones that are affected by invariants.

Lemma 4.11 (Well-definedness). The interpretation in Figure 8 is well-defined:

(1) If the free relation variables of $P$ are contained in $\Xi=X_{1}, \ldots, X_{n}$ then $\llbracket P \rrbracket_{\eta}$ denotes a non-expansive function from $\prod_{X_{i} \in \Xi} \operatorname{Pred}^{\left(\mathrm{Val}^{n}\right)}$ to Pred.

(2) If $P$ is formally contractive in $X$ then the functional $\lambda q \cdot \llbracket P \rrbracket_{\eta, \rho[X:=q]}$ is a contractive map from Pred ${ }^{\left(\mathrm{Val}^{n}\right)}$ to Pred.

Proof sketch. Both parts are proved simultaneously by induction on the structure of $P$. The second part is used to show the well-definedness of recursive specifications, using the fact that the fixed point operator itself is non-expansive. Details are given in Appendix B.2.

As a consequence of the interpretation of triples, the axiom $\{\{A\} e\{B\} \wedge A\} e\{B\}$ does not hold; the inner triple is only approximately valid up to the level of the rank of the argument heap. Similarly, the following rule

$$
\frac{\{A\} e\{B\} \Rightarrow\{P\} e^{\prime}\{Q\}}{\{\{A\} e\{B\} \wedge P\} e^{\prime}\{Q\}}
$$


is not validated by our semantics (the opposite direction actually holds; see Section (5). Axioms and rules like these are used, e.g., by Honda et al. [12, in proofs for recursion through the store; instead we use (EvaL).

4.6. Soundness of the axioms and proof rules. We prove soundness of the axioms and proof rules listed in Sections 2 and 3. We start by defining a notion of validity for judgements and rules with respect to which the soundness will be shown.

Definition 4.12 (Validity of judgements). A judgement $\Xi ; \Gamma \vdash\{P\}^{\prime} C$ ' $\{Q\}$ is valid if, and only if, for all $\eta \in E n v$ such that $\operatorname{dom}(\eta) \supseteq \Gamma$ and for all $\rho \in \prod_{X_{i} \in \Xi} \operatorname{Pred}^{\left(\operatorname{Val}^{n_{i}}\right)}$ such that $n_{i}$ is the arity of $X_{i}$ we have $\models\left\{\llbracket P \rrbracket_{\eta, \rho}\right\} \llbracket C \rrbracket_{\eta}\left\{\llbracket Q \rrbracket_{\eta, \rho}\right\}$. A rule $\frac{J_{1}}{J_{2}}$ is then called sound if validity of judgement $J_{1}$ implies the validity of judgement $J_{2}$. Similarly, an axiom $J$ is called sound if judgement $J$ is valid.

Below we prove the most interesting rules of our logic sound. Where proofs are parametric in the assertions we will directly work with semantic Hoare triples.

Let us first consider the distribution axioms for $-\otimes R$ given in Figure 2 ,

Lemma 4.13 (Distribution axioms). The distribution axioms for $-\otimes R$ are valid.

Proof. We consider the case of invariant extension and triples:

- The validity of $(P \otimes Q) \otimes R \Leftrightarrow P \otimes(Q \circ R)$ is an instance of the fact that $\otimes$ is a monoid action (Lemma 4.5).

- The validity of $\{P\} e\{Q\} \otimes R \Leftrightarrow\{P \circ R\} e\{Q \circ R\}$ follows from the following claim: for all $p, q, r \in$ Pred, strict continuous $c:$ Heap $\multimap T_{\text {err }}($ Heap $)$ and all $w \in W, \iota(r) \circ w \models\{p\} c\{q\}$ if and only if $w \models\{p \otimes \iota(r) * r\} c\{q \otimes \iota(r) * r\}$. The proof of this claim uses the property

$$
\forall p .(p \otimes \iota(r) * r)(w) * \iota^{-1}(w)(e m p)=p(\iota(r) \circ w) * \iota^{-1}(\iota(r) \circ w)(e m p) .
$$

This property is a consequence of the definitions of $\otimes$ and $\circ$ :

$$
\begin{aligned}
(p \otimes \iota(r) * r)(w) * \iota^{-1}(w)(e m p) & =(p \otimes \iota(r))(w) * r(w) * \iota^{-1}(w)(e m p) \\
& =p(\iota(r) \circ w) * r(w \circ e m p) * \iota^{-1}(w)(e m p) \\
& =p(\iota(r) \circ w) *(r \otimes w)(e m p) * \iota^{-1}(w)(e m p) \\
& =p(\iota(r) \circ w) *\left(r \otimes w * \iota^{-1}(w)\right)(e m p) \\
& =p(\iota(r) \circ w) * \iota^{-1}(\iota(r) \circ w)(e m p) .
\end{aligned}
$$

The proofs of the remaining distribution axioms are easy since the logical connectives are interpreted pointwise, and since $e m p$ and $\left(e_{1} \mapsto e_{2}\right)$ are constant.

Next, we consider the proof rules for higher-order store given in Figure 6 .

Lemma 4.14 ( $\otimes$-Frame). The $\otimes$-FrAme rule is sound: if $h \in p(w)$ for all $h \in$ Heap and $w \in W$, then $h \in(p \otimes \iota(r))(w)$ for all $h \in$ Heap, $w \in W$ and $r \in$ Pred.

Proof. Assume that $h \in p(w)$ holds for all $h \in$ Heap and $w \in W$. Let $r \in$ Pred, $w \in W$ and $h \in$ Heap. We show $h \in(p \otimes \iota(r))(w)$. Note that we have $(p \otimes \iota(r))(w)=p(\iota(r) \circ w)$ by the definition of $\otimes$. So, for $w^{\prime} \stackrel{\text { def }}{=} \iota(r) \circ w$, the assumption yields $h \in p\left(w^{\prime}\right)=(p \otimes \iota(r))(w)$. 
The rule $(\otimes-$ MONO), which expresses the monotonicity of $\otimes$ in its left-hand argument, is in fact derivable from $(\otimes$-Frame) and the distribution axioms. Thus, its soundness is a consequence of Lemmas 4.13 and 4.14 ,

Lemma 4.15 (*-Frame). The axiom $\{P\} e\{Q\} \Rightarrow\{P * R\} e\{Q * R\}$ is valid for all $P, Q, R, e$.

Proof. We show that for all worlds $w \in W$, predicates $p, q, r \in$ Pred and commands $c \in C o m$, if $w \models\{p\} c\{q\}$, then $w \models\{p * r\} c\{q * r\}$. This implies the lemma as follows. If $k>0$ is the rank of $\pi_{n}(h)$ and $\pi_{n}(h) \in \llbracket\{P\}$ e $\{Q\} \rrbracket_{\eta, \rho} w$, then $w \models_{k-1}\left\{\llbracket P \rrbracket_{\eta, \rho}\right\} \llbracket e \rrbracket_{\eta}\left\{\llbracket Q \rrbracket_{\eta, \rho}\right\}$. This lets us conclude $w \models_{k-1}\left\{\llbracket P * R \rrbracket_{\eta, \rho}\right\} \llbracket e \rrbracket_{\eta}\left\{\llbracket Q * R \rrbracket_{\eta, \rho}\right\}$, which in turn implies that $\pi_{n}(h)$ is in $\llbracket\{P * R\} e\{Q * R\} \rrbracket_{\eta, \rho} w$.

To prove the claim, assume $w \models\{p\} c\{q\}$. We must show that $w \models\{p * r\} c\{q * r\}$. Let $r^{\prime} \in U A d m$ and assume

$$
h \in(p * r)(w) * \iota^{-1}(w)(e m p) * r^{\prime}=p(w) * \iota^{-1}(w)(e m p) *\left(r(w) * r^{\prime}\right) .
$$

Since $w \models\{p\} c\{q\}$, it follows that

$$
c(h) \in \operatorname{Ad}\left(q(w) * \iota^{-1}(w)(e m p) *\left(r(w) * r^{\prime}\right)\right)=\operatorname{Ad}\left((q * r)(w) * \iota^{-1}(w)(e m p) * r^{\prime}\right),
$$

which establishes $w \models\{p * r\} c\{q * r\}$.

Lemma 4.16 (Eval). Suppose that $R[k] \Rightarrow\{P * e \mapsto R[-]\} k\{Q\}$ is a valid implication. Then, if there are no free occurrences of $k$, also $\{P * e \mapsto R[-]\}$ 'eval [e]' $\{Q\}$ is valid.

Proof. Let $w \in W, \eta \in E n v$ and $r \in U A d m$. Let $\rho$ be a suitable assertion environment. Let $h \in \llbracket P * e \mapsto R[-] \rrbracket_{\eta, \rho} w * \iota^{-1}(w)(e m p) * r$, so that $h=h^{\prime} \cdot h^{\prime \prime}$ for some $h^{\prime}$ and $h^{\prime \prime}$ such that

$$
h^{\prime} \in \llbracket e \mapsto R[-] \rrbracket_{\eta, \rho} w \text { and } h^{\prime \prime} \in \llbracket P \rrbracket_{\eta, \rho} w * \iota^{-1}(w)(e m p) * r .
$$

We must show that $\llbracket$ eval $[e] \rrbracket_{\eta} h \in \operatorname{Ad}\left(\llbracket Q \rrbracket_{\eta, \rho} w * \iota^{-1}(w)(e m p) * r\right)$. Recall that $e \mapsto R[-]$ abbreviates $\exists k . e \mapsto k \wedge R[k]$ for fresh $k$. By (4.2) we have for all $n \geq 0$ such that $\pi_{n}\left(h^{\prime}\right) \neq \perp$ :

$$
\begin{gathered}
\llbracket e \rrbracket_{\eta} \in \operatorname{dom}\left(\pi_{n}\left(h^{\prime}\right)\right)=\operatorname{dom}\left(h^{\prime}\right) \subseteq \operatorname{dom}(h) \\
\exists d_{n} . \pi_{n}(h)\left(\llbracket e \rrbracket_{\eta}\right)=\pi_{n}\left(h^{\prime}\right)\left(\llbracket e \rrbracket_{\eta}\right) \sqsubseteq d_{n} \text { and } \pi_{n}\left(h^{\prime}\right) \in \llbracket R[k] \rrbracket_{\eta\left[k:=d_{n}\right], \rho} w
\end{gathered}
$$

Let us denote $\eta\left[k:=d_{n}\right]$ by $\eta_{n}$. The assumption that $R[k] \Rightarrow\{P * e \mapsto R[-]\} k\{Q\}$ is valid yields:

$$
\pi_{n}\left(h^{\prime}\right) \in \llbracket R[k] \rrbracket_{\eta_{n}, \rho} w \text { implies } \pi_{n}\left(h^{\prime}\right) \in \llbracket\{P * e \mapsto R[-]\} k\{Q\} \rrbracket_{\eta_{n}, \rho} w
$$

Therefore, by (4.4), $\pi_{n}\left(h^{\prime}\right) \in \llbracket\{P * e \mapsto R[-]\} k\{Q\} \rrbracket_{\eta_{n}, \rho} w$ holds for all $n$ sufficiently large. Let $r_{n}$ be the rank of $\pi_{n}\left(h^{\prime}\right)$. Since $\pi_{n}\left(h^{\prime}\right) \neq \perp$ we have $r_{n}>0$. It follows that

$$
\forall n . w \models\left\{\llbracket P * e \mapsto R[-] \rrbracket_{\eta_{n}, \rho}\right\} \pi_{r_{n}-1} ; d_{n} ; \pi_{r_{n}-1}\left\{\llbracket Q \rrbracket_{\eta_{n}, \rho}\right\} .
$$

Since

$$
\pi_{n}\left(h^{\prime}\right)\left(\llbracket e \rrbracket_{\eta}\right)=\pi_{r_{n}}\left(\pi_{n}\left(h^{\prime}\right)\right)\left(\llbracket e \rrbracket_{\eta}\right) \sqsubseteq \pi_{r_{n}-1} ; d_{n} ; \pi_{r_{n}-1},
$$

the downward closure of semantic triples in the command argument gives

$$
\forall n . w \models\left\{\llbracket P * e \mapsto R[-] \rrbracket_{\eta_{n}, \rho}\right\} \pi_{n}\left(h^{\prime}\right)\left(\llbracket e \rrbracket_{\eta}\right)\left\{\llbracket Q \rrbracket_{\eta_{n}, \rho}\right\} .
$$

Since $k$ was chosen fresh, by the admissibility of semantic triples we thus obtain

$$
\left.\forall n . w \models\{\llbracket P * e \mapsto R[]] \rrbracket_{\eta, \rho}\right\} h^{\prime}\left(\llbracket e \rrbracket_{\eta}\right)\left\{\llbracket Q \rrbracket_{\eta, \rho}\right\} .
$$




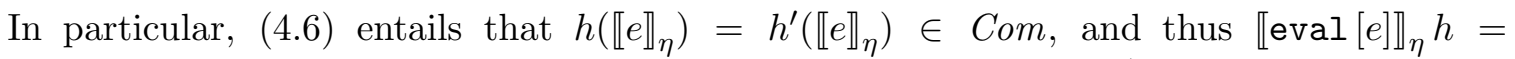
$h^{\prime}\left(\llbracket e \rrbracket_{\eta}\right)(h)$. Since we assumed that $\left.h \in \llbracket P * e \mapsto R[]\right] \rrbracket_{\eta, \rho} w * \iota^{-1}(w)(e m p) * r$, we can conclude $\llbracket$ eval $[e] \rrbracket_{\eta} h \in \operatorname{Ad}\left(\llbracket Q \rrbracket_{\eta, \rho} w * \iota^{-1}(w)(e m p) * r\right)$ by (4.6).

The soundness of the standard Hoare logic rules is straightforward. We illustrate this for the sequencing rule next.

Lemma 4.17 (Sequencing). Provided $\{P\}^{\prime} C^{\prime}\{R\}$ and $\{R\}^{\prime} D^{\prime}\{Q\}$ are valid, then so is $\{P\}^{\prime} C ; D$ ' $\{Q\}$.

Proof. Let $\eta \in E n v, w \in W$, let $\rho$ be an assertion environment, and let $r \in U A d m$. Let $h \in$ $\llbracket P \rrbracket_{\eta, \rho}(w) * \iota^{-1}(w)(e m p) * r$. We must show that $\llbracket C ; D \rrbracket_{\eta} h \in \operatorname{Ad}\left(\llbracket Q \rrbracket_{\eta, \rho}(w) * \iota^{-1}(w)(e m p) *\right.$ $r)$. First note that $\llbracket C \rrbracket_{\eta} h \in \operatorname{Ad}\left(\llbracket R \rrbracket_{\eta, \rho}(w) * \iota^{-1}(w)(e m p) * r\right)$, by the assumption that $\{P\}^{\prime} C^{\prime}\{R\}$ is valid. In particular, $\llbracket C \rrbracket_{\eta} h \neq$ error. Moreover, in the case where $\llbracket C \rrbracket_{\eta} h=$ $\perp$ we also have $\llbracket C ; D \rrbracket_{\eta} h=\perp$ by the semantics of sequential composition, so that the admissibility of $\operatorname{Ad}\left(\llbracket Q \rrbracket_{\eta, \rho}(w) * \iota^{-1}(w)(e m p) * r\right)$ gives the result.

Thus, we can assume that $\llbracket C ; D \rrbracket_{\eta} h=\llbracket D \rrbracket_{\eta}\left(\llbracket C \rrbracket_{\eta} h\right)$. From the assumption that $\{R\}^{\prime} D$ ' $\{Q\}$ is valid it follows that $\llbracket D \rrbracket_{\eta}$ maps the set $\left(\llbracket R \rrbracket_{\eta, \rho}(w) * \iota^{-1}(w)(e m p) * r\right)$ into $\operatorname{Ad}\left(\llbracket Q \rrbracket_{\eta, \rho}(w) * \iota^{-1}(w)(e m p) * r\right)$. Since $\llbracket C \rrbracket_{\eta} h \in \operatorname{Ad}\left(\llbracket R \rrbracket_{\eta, \rho}(w) * \iota^{-1}(w)(e m p) * r\right)$ we obtain $\llbracket D \rrbracket_{\eta}\left(\llbracket C \rrbracket_{\eta} h\right) \in \operatorname{Ad}\left(\llbracket Q \rrbracket_{\eta, \rho}(w) * \iota^{-1}(w)(e m p) * r\right)$ by Lemma 4.7 and continuity of $\llbracket D \rrbracket_{\eta}$.

The proofs for the remaining rules from Figure 3 are similar, and given in Appendix B.3. An exception is the rule of consequence: The soundness proof of rule (CONSEQ) is slightly different from those of the others because (CONSEQ) involves an implication between triples, whereas the other rules are inference rules for transforming valid Hoare triples. Due to the pointwise interpretation of implication and the inclusion of the approximations in the interpretation of triples, this form of the consequence rule could be potentially problematic. Our proof of (CONSEQ) overcomes this potential problem, by exploiting the fact that the rule is "parametric" in the command, i.e., it is the same command that appears in all the triples of the rule. Two further cases that are similar in this respect are the axioms (ExistAux) for the elimination of auxiliary variables and (DISJ); see Appendix B.3.

Lemma 4.18 (Consequence). If $P^{\prime} \Rightarrow P$ and $Q \Rightarrow Q^{\prime}$ are valid implications, then so is $\{P\} e\{Q\} \Rightarrow\left\{P^{\prime}\right\}$ e $\left\{Q^{\prime}\right\}$.

Proof. Let $\eta \in$ Env, $\rho$ an assertion environment, and fix $w \in W$ and $n \geq 0$. Let $p=\llbracket P \rrbracket_{\eta, \rho}$, $p^{\prime}=\llbracket P^{\prime} \rrbracket_{\eta, \rho}, q=\llbracket Q \rrbracket_{\eta, \rho}$ and $q^{\prime}=\llbracket Q^{\prime} \rrbracket_{\eta, \rho}$, and assume that $\pi_{n}(h) \in \llbracket\{P\} e\{Q\} \rrbracket_{\eta} w$. We must prove that $\pi_{n}(h) \in \llbracket\left\{P^{\prime}\right\} e\left\{Q^{\prime}\right\} \rrbracket_{\eta} w$.

Let $k$ denote the rank of $\pi_{n}(h)$. Without loss of generality, we can assume $k>0$. Let $c$ denote the command $\pi_{k-1} ; \llbracket e \rrbracket_{\eta} ; \pi_{k-1}$. Then the assumption yields $w \models\{p\} c\{q\}$, and it suffices to establish $w \models\left\{p^{\prime}\right\} c\left\{q^{\prime}\right\}$. For this, suppose that $r \in U A d m$ and let $h^{\prime} \in p^{\prime}(w) *$ $\iota^{-1}(w)(e m p) * r$. We must show that $c\left(h^{\prime}\right) \in \operatorname{Ad}\left(q^{\prime}(w) * \iota^{-1}(w)(e m p) * r\right)$. By the assumption that $P^{\prime} \Rightarrow P$ is valid, we also have $h^{\prime} \in p(w) * \iota^{-1}(w)(e m p) * r$ by the monotonicity of $*$. By assumption, $c\left(h^{\prime}\right) \in \operatorname{Ad}\left(q(w) * \iota^{-1}(w)(e m p) * r\right)$. By the assumption that $Q \Rightarrow Q^{\prime}$ is valid, and using monotonicity of $*$ and $\operatorname{Ad}(\cdot)$, we obtain $c\left(h^{\prime}\right) \in \operatorname{Ad}\left(q^{\prime}(w) * \iota^{-1}(w)(e m p) * r\right)$ as required. 


\section{Proof Rules involving Different Nesting Levels}

The soundness of rule (EvAL) as shown in Lemma 4.16 involves an assertion $R$ that is used at different nesting levels in its hypothesis and conclusion. In this section we discuss two further proof rules that relate nested triples to top-level implications in a similar way:

$$
\begin{array}{ll}
\text { Out-T } & \Xi ; \Gamma \vdash\{\{A\} d\{B\} \wedge P\} e\{Q\} \\
\Xi ; \Gamma \vdash\{A\} d\{B\} \Rightarrow\{P\} e\{Q\} & \frac{\text { In-T }}{\Xi ; \Gamma \vdash\{A\} d\{B\} \Rightarrow\{P\} e\{Q\}}
\end{array}
$$

While, at first glance, both rules may seem reasonable, we will show below that in our model rule (OUT-T) is valid but rule (IN-T) is not. We begin by making some observations regarding the semantics of nested triples.

Lemma 5.1. For any $w \in W, p, q \in$ Pred and $c \in C o m$ we have that $w \models_{k}\{p\} c\{q\}$ if and only if for all $r \in U A d m, n \leq k$ and all $h \in$ Heap:

$$
\pi_{n}(h) \in p(w) * \iota^{-1}(w)(e m p) * r \Rightarrow \pi_{n}\left(c\left(\pi_{n}(h)\right)\right) \in \operatorname{Ad}\left(q(w) * \iota^{-1}(w)(e m p) * r\right) .
$$

Proof. For the direction from left to right, let $n \leq k$. Using the assumption and $\pi_{n}(h) \in$ $p(w) * \iota^{-1}(w)(e m p) * r$ we obtain $\pi_{k}\left(c\left(\pi_{k}\left(\pi_{n}(h)\right)\right)\right) \in \operatorname{Ad}\left(q(w) * \iota^{-1}(w)(e m p) * r\right)$, thus $\pi_{k}\left(c\left(\pi_{n}(h)\right)\right) \in \operatorname{Ad}\left(q(w) * \iota^{-1}(w)(e m p) * r\right)$ and by downward-closure also $\pi_{n}\left(c\left(\pi_{n}(h)\right)\right) \in$ $\operatorname{Ad}\left(q(w) * \iota^{-1}(w)(e m p) * r\right)$.

For the direction from right to left, let $h \in p(w) * \iota^{-1}(w)(e m p) * r$. By uniformity we know that for all $n \in \omega$ also $\pi_{n}(h) \in p(w) * \iota^{-1}(w)(e m p) * r$. We thus know by assumption that $\pi_{n}\left(c\left(\pi_{n}(h)\right)\right) \in \operatorname{Ad}\left(q(w) * \iota^{-1}(w)(e m p) * r\right)$ for $n \leq k$ and thus in particular for $n=k$.

Definition 5.2. A predicate $p \in$ Pred is pseudo pure if for all $h, h^{\prime} \in$ Heap such that $r n k(h)=r n k\left(h^{\prime}\right)$ and all $w \in W$ we have that $h \in p(w)$ if, and only if, $h^{\prime} \in p(w)$. An assertion is pseudo pure if its denotation is a pseudo pure predicate.

In the following, $\phi$ will always stand for an assertion that is pseudo pure. Note that the typical examples for pseudo pure assertions are triples. Obviously, every pure (i.e., entirely heap-independent) assertion is trivially also pseudo pure. Assertions that depend on the shape and content of the heap itself, e.g. $x \mapsto_{-}$, are not pseudo pure. We also observe that the interpretation of a pseudo pure assertion is downward closed in the rank itself:

Lemma 5.3. For any pseudo pure assertion $p$, and any heaps $h$ and $h^{\prime}$, if $r n k\left(h^{\prime}\right) \leq r n k(h)$ then $h \in p(w)$ implies $h^{\prime} \in p(w)$.

Proof. Suppose $h \in p(w)$, and let $n=r n k\left(h^{\prime}\right) \leq r n k(h)$. Thus we have $r n k\left(\pi_{n}(h)\right)=n$. Since $\pi_{n}(h) \in p(w)$ by uniformity, we can conclude $h^{\prime} \in p(w)$ from the assumption that $p$ is pseudo pure.

With the definition of pseudo pure in place, we can now generalise the rules (OUT-T) and (IN-T) in the following way:

$$
\begin{array}{ll}
\text { Out } & \text { IN } \\
\Xi ; \Gamma \vdash\{\phi \wedge P\} e\{Q\} & \Xi ; \Gamma \vdash \phi \Rightarrow\{P\} e\{Q\}
\end{array}(\phi \text { pseudo pure }) \quad \frac{\Xi ; \Gamma \vdash\{P\} e\{Q\}}{\Xi ; \Gamma \vdash\{\phi \wedge P\} e\{Q\}}(\phi \text { pseudo pure })
$$

Proposition 5.1. The above rule (OUT) is sound.

Proof. Assume environments $\eta$ and $\rho$, let $n \in \omega, w \in W$ and $h \in$ Heap be such that

$$
\pi_{n}(h) \in \llbracket \phi \rrbracket_{\eta, \rho} w
$$


We have to show that $\pi_{n}(h) \in \llbracket\{P\} e\{Q\} \rrbracket_{\eta, \rho} w$. Let $k$ denote the rank of $\pi_{n}(h)$. If $k=0$ we are done. Otherwise we have to show that $w \models_{k-1}\left\{\llbracket P \rrbracket_{\eta, \rho}\right\} \llbracket e \rrbracket_{\eta}\left\{\llbracket Q \rrbracket_{\eta, \rho}\right\}$. But by the observation in Lemma 5.1, it suffices to show for any heap $h^{\prime}$ and any $l \leq k-1$ that if $\pi_{l}\left(h^{\prime}\right) \in \llbracket P \rrbracket_{\eta, \rho}(w) * \iota^{-1}(w)(e m p) * r$ then $\pi_{l}\left(c\left(\pi_{l}\left(h^{\prime}\right)\right)\right) \in \operatorname{Ad}\left(\llbracket Q \rrbracket_{\eta, \rho}(w) * \iota^{-1}(w)(e m p) * r\right)$. From the interpretation of the premise of the rule using $\eta, w$ and $l$ we get the desired result if we can show that $\pi_{l}\left(h^{\prime}\right) \in \llbracket P \rrbracket_{\eta, \rho}(w) * \iota^{-1}(w)(e m p) * r$ implies $\pi_{l}\left(h^{\prime}\right) \in \llbracket \phi \wedge P \rrbracket_{\eta, \rho}(w) *$ $\iota^{-1}(w)(e m p) * r$. Yet, $\pi_{l}\left(h^{\prime}\right) \in \llbracket \phi \rrbracket_{\eta, \rho}(w)$ follows from Lemma 5.3 due to assumption (5.1), the fact that $\phi$ is pseudo pure, and the fact that $r n k\left(\pi_{l}\left(h^{\prime}\right)\right) \leq l<k=r n k\left(\pi_{n}(h)\right)$.

Proposition 5.2. The rule (IN) does not hold in our model.

Proof. Assuming that (IN) holds in our semantics we can derive an invalid triple as follows. Let $R$ abbreviate the recursive assertion $\mu X .\{X\}$ 'skip' $\{$ false $\}$. Then, from the tautology $R \Rightarrow R$ we obtain $R \Rightarrow\{R\}$ 'skip' \{false $\}$ by unfolding the recursive definition of $R$. Applying (IN) and the consequence rule thus gives

$$
\vdash\{R\} \text { 'skip' }\{\text { false }\}
$$

Our model validates the implication $e m p \Rightarrow R$ : By definition of implication, it suffices to prove that $\operatorname{rnk}(\{\mid\})=n$ implies $w \models_{n-1}\{\llbracket R \rrbracket\} \llbracket$ skip $\rrbracket\{\llbracket$ false $\rrbracket\}$. Since the empty heap has rank 1, this implication holds trivially for any triple on the right hand side, in particular $R$. From (5.2) and this implication we conclude that the triple $\{e m p\}$ 'skip' $\{$ false $\}$ holds, which is clearly not the case by definition of the semantics of triples. We conclude that rule (IN) cannot hold with respect to our semantics.

It is worth looking more closely at the reason why rule (IN) does not hold in our semantics. Essentially, to show the triple in the conclusion at level $k$, one needs to show that in the hypothesis the formula $\phi$ holds for a heap with rank $k+1$. But this property cannot be established in general from the assumptions of the triple in the conclusion at level $k 9$ Note that, in the case of (EVAL), the corresponding property can be established since the heap access of the eval command offsets the increase in the rank (cf. equation (4.5) in the proof of Lemma 4.16).

In the case where the command is arbitrary (i.e., not eval), one can express the upwards shift of levels explicitly with the help of a modal operator $\diamond P$ ("previous $P$," or " $P$ one level up"). This operator is defined by $h \in \llbracket \triangleright P \rrbracket_{\eta, \rho} w$ if and only if

- $\operatorname{rnk}(h)=\infty$ and $h \in \llbracket P \rrbracket_{\eta, \rho} w$ or

- $r n k(h)=k<\infty$ and there exists $h^{\prime} \in \llbracket P \rrbracket_{\eta, \rho} w$ such that $r n k\left(h^{\prime}\right)=k+1$ and $\pi_{k}\left(h^{\prime}\right)=h$, and thus $\diamond P$ denotes a downward closed, admissible predicate. With the help of the modality, we can give variants of the above rules that keep track of the rank information:

$$
\begin{aligned}
& \diamond \text { OUT } \quad \diamond \mathrm{IN} \quad \diamond \mathrm{E} \\
& \frac{\Xi ; \Gamma \vdash\{\phi \wedge P\} e\{Q\}}{\Xi ; \Gamma \vdash \phi \Rightarrow \diamond\{P\} e\{Q\}} \quad \frac{\Xi ; \Gamma \vdash \phi \Rightarrow \diamond\{P\} e\{Q\}}{\Xi ; \Gamma \vdash\{\phi \wedge P\} e\{Q\}} \quad \overline{\Xi ; \Gamma \vdash \diamond P \Rightarrow P}
\end{aligned}
$$

In our semantics, which still satisfies $(\diamond \mathrm{OUT})$ and $(\diamond \mathrm{E})$, even this strengthened variant $(\diamond \mathrm{IN})$ does not hold. This is due to the following simple observation, which means that ranks are not preserved by the separating conjunction that is used in the interpretation of triples.

\footnotetext{
${ }^{9}$ Rule (IN) does hold in the special case when $\phi$ is pure.
} 
Lemma 5.4. Given a heap $h=h_{1} \cdot h_{2}$ with rank $n$, such that $r n k\left(h_{1}\right)=n_{1}$ and $r n k\left(h_{2}\right)=n_{2}$ it may well be the case that $n_{1}<n$ or $n_{2}<n$.

However, the modal rules can be proved sound with the help of a step-indexed model. In such a model, the ranks are replaced by an explicit natural number index that gives a lower bound on the number of steps that can be safely taken in an operational semantics without invalidating a given assertion. The slightly unintuitive implications emp $\Rightarrow\{P\} e\{Q\}$ will not hold in the step-indexed model either. However, also the step-indexed model does not validate (IN), and we conjecture that this rule renders the logic inconsistent. More details about step-indexed models can be found in [4].

It is worth pointing out that not only unintuitive implications emp $\Rightarrow\{P\} e\{Q\}$ do hold in our model, but also that the so-called invariance rull10

$$
\begin{aligned}
& \text { INVARIANCE } \\
& \Xi ; \Gamma \vdash\{P\} e\{Q\} \\
& \Xi ; \Gamma \vdash\{P \wedge\{A\} k\{B\}\} e\{Q \wedge\{A\} k\{B\}\}
\end{aligned}
$$

does not hold. It is only valid for invariants that are pure, so it does not hold for $\{A\} k\{B\}$ nor any other pseudo pure invariant. This can be easily seen by considering the triple

$$
\{e m p\} \text { 'let } x=\text { new } 0 \text { in }[x]:=\text { 'skip' ' }\{\exists x . x \mapsto\{e m p\}-\{e m p\}\}
$$

with invariant $\{e m p\}$ skip $\{$ false $\}$, since the latter only holds for heaps with rank 1 , for instance the empty heap. Unfortunately, not even the following restricted form of invariance holds:

$$
\begin{aligned}
& \text { INVARIANCER } \\
& \frac{\Xi ; \Gamma \vdash\left\{P * e_{1} \mapsto e_{2}\right\} e\left\{Q * e_{1} \mapsto e_{2}\right\}}{\Xi ; \Gamma \vdash\left\{P *\left(e_{1} \mapsto e_{2} \wedge \phi\right)\right\} e\left\{Q *\left(e_{1} \mapsto e_{2} \wedge \phi\right)\right\}}(\phi \text { pseudo pure })
\end{aligned}
$$

since the semantics of triples and of $\mapsto$ does not guarantee that the data stored at $\llbracket e_{1} \rrbracket$, and thus the rank of any heap fulfilling $\llbracket e_{1} \mapsto e_{2} \wedge \phi \rrbracket$, is invariant. It could still be the case that the result heap meeting the postcondition $\llbracket e_{1} \mapsto e_{2} \wedge \phi \rrbracket$ has a higher rank than the pre-execution heap meeting the same condition. The only way to guarantee that invariance involving triples or other pseudo pure assertions holds is to ensure that the rank (or even the content) of the heap cells in question does not change during execution. Because of this issue we needed another update rule for programs that copy code:

$$
\overline{\Xi ; \Gamma \vdash\left\{e \mapsto{ }_{-} *\left(e_{1} \mapsto e_{0} \wedge \phi\right)\right\} '[e]:=e_{0}^{\prime}\left\{\left(e \mapsto e_{0} \wedge \phi\right) *\left(e_{1} \mapsto e_{0} \wedge \phi\right)\right\}}(\phi \text { pseudo pure })
$$

Note that $\phi$ may contain the expression $e_{0}$ (which can also be a variable) and will typically be a triple $\{A\} k\{B\}$. The soundness of this rule follows from the soundness of the assignment rule (UPDATE) and the fact that the rank of the heap with domain $\llbracket e_{1} \rrbracket$ is not changed by the command. Consequently, the heap with domain $\llbracket e \rrbracket$, which is identical to the one with domain $\llbracket e_{1} \rrbracket$ after execution, satisfies $\phi$. But this axiom is not derivable from (UPDATE) as, for a pseudo pure $\phi$, the axiom

$$
e \mapsto e_{0} *\left(e_{1} \mapsto e_{0} \wedge \phi\right) \Rightarrow\left(e \mapsto e_{0} \wedge \phi\right) *\left(e_{1} \mapsto e_{0} \wedge \phi\right)
$$

does not hold for the same reasons as (INVARIANCER) does not hold.

\footnotetext{
${ }^{10}$ This should not be confused with the stronger conjunction rule which is known to be inconsistent with higher-order frame rules [16]).
} 


\section{Conclusion}

In this article we have investigated a separation logic for a simple programming language with higher-order store. As our counterexamples illustrate, the design of such a logic is not straightforward:

- In the presence of recursive assertions, unrestricted use of a deep frame axiom permits the "laundering" of code, which allows for the derivation of insufficient memory footprints (Proposition 3.1).

- Higher-order frame rules are inconsistent with a classical specification logic (and hence in our case, due to the identification of assertion and specification language, with a classical assertion logic; Proposition 3.3.

- In the presence of recursive assertions, one cannot move global assumptions of triples, expressed as implications, into pre-conditions (Proposition [5.2).

Note that the first two points are independent of any choice of model whereas this is not clear for the third point.

In our model, we use recursively defined Kripke worlds to interpret the invariant extension $P \otimes R$. In a logic without recursive assertions (and assertion variables), like the one considered by Birkedal et al. for Idealized Algol [7], the invariant extension operation can be considered essentially as a syntactic abbreviation. In particular, it need not be treated as a primitive operation and recursive worlds are not needed. In a logic with second-order quantification, frame conditions can be made explicit in a specification, which gives rise to a modular proof pattern without explicit deep frame rule; this idea is discussed and used in, e.g., [3, 10].

Recursive worlds similar to the ones employed here can be used to construct a model for Pottier's anti-frame rule, a proof rule for hiding local state from the context [20]. In that case, predicates must depend on the worlds in a monotonic way (with respect to an order on worlds defined from the composition operation $\circ$ ), which complicates the model construction considerably [28, 27].

During the process of writing this article, it has been discovered that one can also build a model for the presented logic, including deep frame rules and recursive assertions, with the help of step-indexing [2] based on an operational semantics for the programming language. We have already pointed out differences regarding both models throughout the paper but here is a short summary. The domain model in our work uses ranks of heaps in order to equip semantic assertions with an ultrametric. Whereas steps are counted separately in the step-indexed approach, heaps, and thus their ranks, are manipulated by programs. This leads to some contamination of the assertion semantics that the step-index model does not share. First of all, we do not get a BI algebra, more precisely we do not get spatial implication. Secondly, triples are not pure but pseudo pure. This, in turn, means that the invariance rule for triples is not valid and holds only for programs that do not change the rank of the heap in question (as expressed in (UPDATEINV)). Moreover, some unwanted implications between triples are validated. The (IN-T) rule does not hold in either of the two models but it holds in [12]. The $(\diamond \mathrm{IN})$ rule, on the other hand, does hold in the stepindexed model but not the presented one. Despite the complications caused by the ranks of heaps, the denotational model has some upsides as well. From earlier work one knows that it represents a way to combine some equational reasoning with Hoare style logics. Equational reasoning has been used to some extent to prove properties of the model, in particular the soundness of the presented rules. It remains to be seen whether the denotational models 
have more advantages over the operational step-indexed ones regarding binary relations, e.g. in order to prove parametricity results.

A detailed description of the step-indexed model and its applications will appear elsewhere in due course.

The work of Honda et al. 12] also presents a logic for higher-order functions and general references, including even observational completeness, i.e. two programs are equal if they fulfill the same triples. The main differences with respect to the logic presented here are as follows. In 32 a logic for total correctness is given. Therefore, there is no need for a specific rule handling recursion through the store, since procedures are always proved sound using induction on a termination measure that the verifier needs to guess. Moreover, local reasoning is ignored so there are no frame rules. The follow-up work [32] addressed this issue, but using content quantification and not separation logic. There does not appear to be an implementation of the logic of [32] either.

A variant of our logic, for a language with recursive procedures and the possibility of partial application, has been implemented in the Crowfoot tool [9]. This verification tool is mainly targeted to prove memory safety for programs with stored procedures automatically. In its current state it does not yet cover a full-fledged first-order logic. Some example specifications for nested triples and recursive assertions can be found e.g. in [11].

\section{ACKNOWLEDGMent}

We would like to thank Nathaniel "Billiejoe" Charlton, François Pottier, Kristian Støvring and Jacob Thamsborg for helpful discussions. Kristian suggested that $\otimes$ is a monoid action. We are grateful for the suggestions of the anonymous referees to improve the paper. Partial support has been provided by FNU project 272-07-0305 "Modular reasoning about software" (Birkedal), EPSRC projects EP/G003173/1 "From reasoning principles for function pointers to logics for self-configuring programs" (Reus), EP/E053041/1 "Scalable program analysis for software verification" and EP/H008373/1 "Resource reasoning" (Yang).

\section{REFERENCES}

[1] P. America and J. J. M. M. Rutten. Solving reflexive domain equations in a category of complete metric spaces. J. Comput. Syst. Sci., 39(3):343-375, 1989.

[2] A. W. Appel and D. A. McAllester. An indexed model of recursive types for foundational proof-carrying code. ACM Trans. Program. Lang. Syst., 23(5):657-683, 2001.

[3] N. Benton. Abstracting allocation. In Proceedings of CSL, pages 182-196, 2006.

[4] L. Birkedal, B. Reus, J. Schwinghammer, K. Støvring, J. Thamsborg, and H. Yang. Step-indexed Kripke models over recursive worlds. In Proceedings of POPL, pages 119-132, 2011.

[5] L. Birkedal, B. Reus, J. Schwinghammer, and H. Yang. A simple model of separation logic for higherorder store. In Proceedings of ICALP, pages 348-360, 2008.

[6] L. Birkedal, K. Støvring, and J. Thamsborg. Realizability semantics of parametric polymorphism, general references, and recursive types. In Proceedings of FOSSACS, pages 456-470, 2009.

[7] L. Birkedal, N. Torp-Smith, and H. Yang. Semantics of separation-logic typing and higher-order frame rules for Algol-like languages. Logical Methods in Computer Science, 2(5:1), 2006.

[8] L. Birkedal and H. Yang. Relational parametricity and separation logic. Logical Methods in Computer Science, 4(2:6), 2008.

[9] N. Charlton, B. Horsfall, and B. Reus. Crowfoot: a verifier for higher order store programs. Unpublished, available at http://www.informatics.sussex.ac.uk/research/projects/PL4HOStore/crowfoot, Feb. 2011. 
[10] N. Charlton and B. Reus. A deeper understanding of the deep frame axiom (extended abstract). Presented at LOLA Workshop 2010 (Syntax and Semantics of Low Level Languages), July 2010.

[11] N. Charlton and B. Reus. Specification patterns and proofs for recursion through the store. In Proceedings of FCT, 2011.

[12] K. Honda, N. Yoshida, and M. Berger. An observationally complete program logic for imperative higherorder functions. In Proceedings of LICS, pages 270-279, 2005.

[13] N. Krishnaswami, L. Birkedal, J. Aldrich, and J. C. Reynolds. Idealized ML and Its Separation Logic. Available at http://www.cs.cmu.edu/ neelk/, 2007.

[14] A. Nanevski, G. Morrisett, A. Shinnar, P. Govereau, and L. Birkedal. Ynot: dependent types for imperative programs. In Proceedings of ICFP, pages 229-240, 2008.

[15] P. W. O'Hearn and D. J. Pym. The logic of bunched implications. B. Symb. Log., 5(2):215-244, 1999.

[16] P. W. O'Hearn, H. Yang, and J. C. Reynolds. Separation and information hiding. In Proceedings of POPL, pages 268-280, 2004.

[17] M. Parkinson and G. Biermann. Separation logic, abstraction and inheritance. In Proceedings of POPL, pages 75-86, 2008.

[18] B. C. Pierce. Types and Programming Languages. MIT Press, 2002.

[19] A. M. Pitts. Relational properties of domains. Inf. Comput., 127:66-90, 1996.

[20] F. Pottier. Hiding local state in direct style: a higher-order anti-frame rule. In Proceedings of LICS, pages 331-340, 2008.

[21] F. Pottier. Three comments on the anti-frame rule. Unpublished, July 2009.

[22] D. J. Pym, P. W. O'Hearn, and H. Yang. Possible worlds and resources: the semantics of BI. Theor. Comput. Sci., 315(1):257-305, May 2004.

[23] B. Reus and J. Schwinghammer. Separation logic for higher-order store. In Proceedings of CSL, pages 575-590, 2006.

[24] J. C. Reynolds. Idealized Algol and its specification logic. In D. Néel, editor, Tools and Notions for Program Construction, pages 121-161. Cambridge University Press, 1982.

[25] J. C. Reynolds. Separation logic: A logic for shared mutable data structures. In Proceedings of LICS, pages 55-74, 2002.

[26] J. Schwinghammer, L. Birkedal, B. Reus, and H. Yang. Nested Hoare triples and frame rules for higherorder store. In Proceedings of CSL, pages 440-454, 2009.

[27] J. Schwinghammer, L. Birkedal, and K. Støvring. A step-indexed Kripke model of hidden state via recursive properties on recursively defined metric spaces. In Proceedings of FOSSACS, pages 305-319, 2011.

[28] J. Schwinghammer, H. Yang, L. Birkedal, F. Pottier, and B. Reus. A semantic foundation for hidden state. In Proceedings of FOSSACS, pages 2-17, 2010.

[29] M. B. Smyth and G. D. Plotkin. The category-theoretic solution of recursive domain equations. SIAM J. Comput., 11(4):761-783, 1982.

[30] M. H. Sørensen and P. Urzyczyn. Lectures on the Curry-Howard Isomorphism, volume 149 of Studies in Logic and the Foundations of Mathematics. Elsevier, 2006.

[31] T. Streicher. Domain-theoretic Foundations of Functional Programming. World Scientific, 2006.

[32] N. Yoshida, K. Honda, and M. Berger. Logical reasoning for higher-order functions with local state. In Foundations of Software Science and Computation Structure, pages 361-377, 2007. 


\section{Appendix A. Summary of Proof Rules}

Figure 9 summarizes the proof rules that we have proved sound with respect to our model. Not shown are the standard proof rules for (intuitionistic) first-order logic (for instance, see [30]) and the distribution axioms for $\otimes$ that appear in Figure 2.

\section{Appendix B. Proofs}

This section contains the proofs omitted from the main part of the paper.

\section{B.1. Heyting algebra structure of uniform admissible subsets.}

Lemma B.1 (Heyting algebra). Let $I=\{\{\mid\}, \perp\}$. Then $(U A d m, \subseteq)$ is a complete Heyting algebra with a (monotone) commutative monoid structure $(U A d m, *, I)$. All the algebra operations are non-expansive with respect to the metric defined in Section 4.3.

Proof. Since admissibility and uniformity are preserved by taking arbitrary intersections, $U A d m$ is a complete lattice, with meets given by set-theoretic intersection, least element $\{\perp\}$ and greatest element Heap. Binary joins are given by set-theoretic union, and arbitrary joins by $\bigsqcup_{i} p_{i}=\bigcap\left\{p \in U A d m \mid p \supseteq \bigcup_{i} p_{i}\right\}$.

The join is described more explicitly as $\bigsqcup_{i} p_{i}=\left\{h \mid \forall n \in \omega . \pi_{n}(h) \in \bigcup_{i} p_{i}\right\}$. First, note that the right hand side $r \stackrel{\text { def }}{=}\left\{h \mid \forall n \in \omega . \pi_{n}(h) \in \bigcup_{i} p_{i}\right\}$ is an element of UAdm: $r$ is uniform, i.e., $h \in r$ implies $\pi_{m}(h) \in r$ for all $m \in \omega$, since $\pi_{n} \cdot \pi_{m}=\pi_{\min \{n, m\}}$. To show that $r$ is also admissible suppose $h_{0} \sqsubseteq h_{1} \sqsubseteq \ldots$ is a chain in $r$, and let $h$ be the lub of this chain. We must show that $\pi_{n}(h) \in \bigcup_{i} p_{i}$ for all $n \in \omega$. By compactness, $\pi_{n}(h) \sqsubseteq h_{k} \sqsubseteq h$ for some $k$, and hence $\pi_{n}(h)=\pi_{n}\left(h_{k}\right) \in \bigcup p_{i}$ using the idempotency of $\pi_{n}$ and the fact that $h_{k} \in r$. To see the inclusion $r \subseteq \bigsqcup_{i} p_{i}$, note that for all $h$, if $\pi_{n}(h) \in \bigcup_{i} p_{i} \subseteq p$ for all $n \in \omega$ and some arbitrary $p \in U A d m$, then also $h=\sqcup_{n} \pi_{n}(h) \in p$ by admissibility, and hence $h \in \bigsqcup_{i} p_{i}$ follows. For the other inclusion, we claim that the right hand side $r \stackrel{\text { def }}{=}\left\{h \mid \forall n \in \omega . \pi_{n}(h) \in \bigcup_{i} p_{i}\right\}$ is one of the elements appearing in the intersection; from this claim it is immediate that $r \supseteq \bigsqcup_{i} p_{i}$. The claim follows since $r \supseteq \bigcup_{i} p_{i}$ by the uniformity of the $p_{i}$ 's.

The implication of this complete lattice $U A d m$ is described by $p \Rightarrow q \stackrel{\text { def }}{=}\{h \mid \forall n \in$ $\omega$. if $\pi_{n}(h) \in p$ then $\left.\pi_{n}(h) \in q\right\}$ : Using $\pi_{n} \cdot \pi_{m}=\pi_{\min \{n, m\}}$ it is easy to see that $p \Rightarrow q$ is uniform. Admissibility follows analogously to the case of joins: if $h_{0} \sqsubseteq h_{1} \sqsubseteq \ldots$ is a chain in $p \Rightarrow q$ with lub $h$, and if $n \in \omega$ is such that $\pi_{n}(h) \in p$ then we must show that $\pi_{n}(h) \in q$. Since $\pi_{n}(h) \sqsubseteq h$ is compact, there is some $k$ such that $\pi_{n}(h) \sqsubseteq h_{k} \sqsubseteq h$, and thus the required $\pi_{n}(h)=\pi_{n}\left(h_{k}\right) \in q$ follows from $h_{k} \in p \Rightarrow q$. Next, to see that $p \Rightarrow q$ is indeed the implication in $U A d m$, first note that we have $p \cap(p \Rightarrow q) \subseteq q$, using the uniformity of $p$ and the admissibility of $q$. If $p \cap r \subseteq q$ for some $r \in U A d m$, and $h \in r$ and $\pi_{n}(h) \in p$ for some $n \in \omega$, then the uniformity of $r$ yields $\pi_{n}(h) \in q$. Thus we obtain $p \cap r \subseteq q \Leftrightarrow r \subseteq p \Rightarrow q$.

That $*$ is an operation on $U A d m$ is established in the proof of Lemma 4.3. It is easy to check that $*$ is commutative and associative and that it is monotone, i.e., if $p \subseteq p^{\prime}$ and $q \subseteq q^{\prime}$ then $p * q \subseteq p^{\prime} * q^{\prime}$. Moreover, we have $I \in U A d m$, and the fact that $p * I=p=I * p$ follows from the definition of the heap combination $h \cdot h^{\prime}$.

For the non-expansiveness of the algebra operations, we only consider the case of meets as an example. Assume $p \stackrel{n}{=} p^{\prime}$ and $q \stackrel{n}{=} q^{\prime}$, then whenever $h \in p \cap q$ we have $\pi_{n}(h) \in p^{\prime}$ and $\pi_{n}(h) \in q^{\prime}$ by assumption. Thus also $p \cap q \stackrel{n}{=} p^{\prime} \cap q^{\prime}$. 


\begin{tabular}{|c|c|}
\hline$*$-AssoC & $\Xi ; \Gamma \vdash P *(Q * R) \Leftrightarrow(P * Q) * R$ \\
\hline *-COMM & $\Xi ; \Gamma \vdash P * Q \Leftrightarrow Q * P$ \\
\hline *-UNIT & $\Xi ; \Gamma \vdash P * e m p \Leftrightarrow P$ \\
\hline$*-\mathrm{ZERO}$ & $\Xi ; \Gamma \vdash P *$ false $\Leftrightarrow$ false \\
\hline *-OVERLAP & $\Xi ; \Gamma \vdash\left(e \mapsto e_{1} * e \mapsto e_{2}\right) \Leftrightarrow$ false \\
\hline \multirow{2}{*}{$*-\mathrm{MONO}$} & $\Xi ; \Gamma \vdash P \Rightarrow P^{\prime} \quad \Xi ; \Gamma \vdash Q \Rightarrow Q^{\prime}$ \\
\hline & $\Xi ; \Gamma \vdash P * Q \Rightarrow P^{\prime} * Q^{\prime}$ \\
\hline \multirow{2}{*}{$\otimes-$ MONO } & $\Xi ; \Gamma \vdash P \Rightarrow P^{\prime}$ \\
\hline & $\overline{\Xi ; \Gamma \vdash P \otimes R \Rightarrow P^{\prime} \otimes R}$ \\
\hline \multirow{2}{*}{ DEREF } & 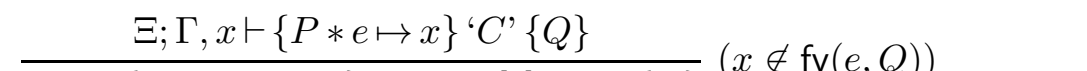 \\
\hline & $\Xi ; \Gamma \vdash\{\exists x . P * e \mapsto x\}{ }^{\prime}$ let $x=[e]$ in $C^{\prime}\{Q\}$ \\
\hline UPDATE & $\Xi ; \Gamma \vdash\left\{e \mapsto \mapsto_{-} * P\right\}^{\prime}[e]:=e_{0}{ }^{\prime}\left\{e \mapsto e_{0} * P\right\}$ \\
\hline \multirow[t]{2}{*}{ UPDATEINV } & $(\phi$ pseudo pure $)$ \\
\hline & $\Xi ; \Gamma \vdash\left\{e \mapsto{ }_{-} *\left(e_{1} \mapsto e_{0} \wedge \phi\right)\right\}^{6}[e]:=e_{0}^{\prime}\left\{\left(e \mapsto e_{0} \wedge \phi\right) *\left(e_{1} \mapsto e_{0} \wedge \phi\right)\right\}$ \\
\hline NEW & $\Xi ; \Gamma, x \vdash\{P * x \mapsto e\}^{\prime} C^{\prime}\{Q\} \quad(x \notin \mathrm{fv}(P, e, Q))$ \\
\hline & $\overline{\Xi ; \Gamma \vdash\{P\} ' \text { let } x=\text { new } e \text { in } C^{\prime}\{Q\}}(x<$ (v $(1, e, Q)$ \\
\hline FREE & $\Xi ; \Gamma \vdash\left\{e \mapsto_{-} * P\right\}^{\prime} \operatorname{free}(e)^{\prime}\{P\}$ \\
\hline \multirow{2}{*}{$\mathrm{IF}$} & $\Xi ; \Gamma \vdash\left\{P \wedge e_{0}=e_{1}\right\}^{\prime} C^{\prime}\{Q\} \quad \Xi ; \Gamma \vdash\left\{P \wedge e_{0} \neq e_{1}\right\}{ }^{\prime} D$ ' $\{Q\}$ \\
\hline & $\Xi ; \Gamma \vdash\{P\}^{\prime}$ if $\left(e_{0}=e_{1}\right)$ then $C$ else $D$ ' $\{Q\}$ \\
\hline SKIP & $\Xi ; \Gamma \vdash\{P\}$ 'skip' $\{P\}$ \\
\hline \multirow{2}{*}{ SEQ } & $\Xi ; \Gamma \vdash\{P\}^{\prime} C^{\prime}\{R\} \quad \Gamma \vdash\{R\}^{\prime} D^{\prime}\{Q\}$ \\
\hline & $\Xi ; \Gamma \vdash\{P\}^{\prime} C ; D^{\prime}\{Q\}$ \\
\hline \multirow{2}{*}{ EVAL } & $\Xi ; \Gamma, k \vdash R[k] \Rightarrow\{P * e \mapsto R[-]\} k\{Q\}$ \\
\hline & $\Xi ; \Gamma \vdash\{P * e \mapsto R[-]\}$ 'eval $[e]$ ' $\{Q\}$ \\
\hline \multirow{2}{*}{ CONSEQ } & $\Xi ; \Gamma \vdash P^{\prime} \Rightarrow P \quad \Xi ; \Gamma \vdash Q \Rightarrow Q^{\prime}$ \\
\hline & $\overline{\Xi ; \Gamma \vdash\{P\} e\{Q\} \Rightarrow\left\{P^{\prime}\right\} e\left\{Q^{\prime}\right\}}$ \\
\hline DisJ & $\Xi ; \Gamma \vdash\left(\{P\} e\{Q\} \wedge\left\{P^{\prime}\right\}\right.$ e $\left.\left\{Q^{\prime}\right\}\right) \Rightarrow\left\{P \vee P^{\prime}\right\} e\left\{Q \vee Q^{\prime}\right\}$ \\
\hline
\end{tabular}

Figure 9. Axioms and proof rules. Rule $\otimes$-Mono is in fact a derived rule.

Lemma B.2 (Heyting algebra, II). The set of non-expansive functions $W \rightarrow U A d m$, ordered pointwise, forms a complete Heyting algebra with a (monotone) commutative monoid 


\begin{tabular}{ll}
\hline Existaux & $\Xi ; \Gamma \vdash(\forall x .\{P\} e\{Q\}) \Rightarrow\{\exists x . P\} e\{\exists x . Q\} \quad(x \notin \mathrm{fv}(e))$ \\
Invariance & $\Xi ; \Gamma \vdash\{P\} e\{Q\} \Rightarrow\{P \wedge \psi\} e\{Q \wedge \psi\} \quad(\psi$ is pure $)$ \\
Q-Frame & $\frac{\Xi ; \Gamma \vdash P}{\Xi ; \Gamma \vdash P \otimes R}$ \\
*-Frame & $\Xi ; \Gamma \vdash\{P\} e\{Q\} \Rightarrow\{P * R\} e\{Q * R\}$ \\
& $\frac{\Xi ; \Gamma \vdash R \Leftrightarrow P[X:=R] \quad \Xi ; \Gamma \vdash S \Leftrightarrow P[X:=S]}{\Xi ; \Gamma \vdash R \Leftrightarrow S}(P$ formally contr. in $X)$
\end{tabular}

Figure 9. Axioms and proof rules (cont.).

structure. The operations are given by the pointwise extension of the corresponding ones on $U A d m$, and they are non-expansive with respect to the sup-metric on $W \rightarrow U A d m$.

Proof. We begin by showing that all the claimed algebra operations on $W \rightarrow U A d m$ are well-defined, i.e., that the pointwise definitions give rise to non-expansive functions from $W$ to $U A d m$. The cases of the various units are given by constant functions and thus non-expansive:

$$
\top(w)=\text { Heap } \quad \perp(w)=\{\perp\} \quad I(w)=\{\{\mid\}, \perp\}
$$

Next, consider the case of meets. Let $\left(p_{i}\right)_{i \in I}$ be a family of functions $p_{i}$ in $W \rightarrow U A d m$ and $w, w^{\prime} \in W$ such that $w \stackrel{n}{=} w^{\prime}$, we have

$$
\left(\bigcap_{i \in I} p_{i}\right)(w)=\bigcap_{i \in I} p_{i}(w) \stackrel{n}{=} \bigcap_{i \in I} p_{i}\left(w^{\prime}\right)=\left(\prod_{i \in I} p_{i}\right)\left(w^{\prime}\right)
$$

by the non-expansiveness of each $p_{i}$. Well-definedness for the other operations is shown analogously.

We now show that the operations are non-expansive. Again, we consider the case of meets only, as the remaining cases are similar. Let $\left(p_{i}\right)_{i \in I}$ and $\left(q_{i}\right)_{i \in I}$ be two families of non-expansive functions such that $p_{i} \stackrel{n}{=} q_{i}$ holds for all $i \in I$. To see that $\prod_{i} p_{i} \stackrel{n}{=} \prod_{i} q_{i}$ holds, by definition of the sup-metric it suffices to prove $\left(\prod_{i} p_{i}\right)(w) \stackrel{n}{=}\left(\prod_{i} q_{i}\right)(w)$ for all $w \in W$. This follows from the pointwise definition since $p_{i}(w) \stackrel{n}{=} q_{i}(w)$ holds for every $i \in I$ by assumption.

\section{B.2. Interpretation of assertions.}

Lemma B.3 (Non-expansiveness of fix, [6]). Let $(X, d)$ be an object in CBUlt, and let $f, g: X \rightarrow X$ be contractive functions on $X$. Then $d(f i x f, f i x g) \leq \sup _{x \in X} d(f(x), g(x))$.

Lemma B.4 (Well-definedness). The interpretation in Fig. 8 is well-defined. More precisely, let $P$ be an assertion with free relation variables in $\Xi=X_{1}, \ldots, X_{k}$, where the arity of $X_{i}$ is $n_{i}$. Then:

(1) for every $\eta \in V a l^{V a r}$ and $\rho \in \prod_{X_{i} \in \Xi} \operatorname{Pred}^{\left(\operatorname{Val}^{n_{i}}\right)}, \llbracket P \rrbracket_{\eta, \rho}$ is an element of Pred, i.e., a non-expansive function $W \rightarrow U A d m$; 
(2) $\llbracket P \rrbracket_{\eta}$ denotes a non-expansive function from $\prod_{X_{i} \in \Xi} \operatorname{Pred}^{\left(\mathrm{Val}^{n_{i}}\right)}$ to Pred;

(3) If $P$ is formally contractive in $X$ then the functional $\lambda q \cdot \llbracket P \rrbracket_{\eta, \rho[X:=q]}$ is a contractive map from Pred ${ }^{\left(\mathrm{Val}^{n}\right)}$ to Pred, where $X$ is an $n$-ary relation variable.

Proof. The claims are proved simultaneously by induction on the structure of $P$. Note that the composition of non-expansive functions is again a non-expansive function, and that the composition of a contractive function with a non-expansive function is again a contractive function.

- For the logical connectives, the claims follow from the inductive hypothesis and Lemmas B.1 and B.2 respectively.

- The case of invariant extension, $P \otimes R$, follows from Lemma 4.4. In particular, $q \mapsto$ $\llbracket P \otimes R \rrbracket_{\eta, \rho[X:=q]}$ is a contractive function whenever $P$ is formally contractive in $X$.

- The case of a relation variable, $X_{i}(\vec{e})$, follows from the assumption that $\rho\left(X_{i}\right)$ is a nonexpansive function from $V a l^{n_{i}}$ to Pred.

- In the case of recursive assertions, $(\mu X(\vec{x}) \cdot P)(\vec{e})$, the well-formedness requirement that $P$ be formally contractive in $X$ means that $\lambda q . \llbracket P \rrbracket_{\eta, \rho[X:=q]}$ is contractive, by part (3) of the induction hypothesis. Hence, $\lambda q, \vec{d} . \llbracket P \rrbracket_{\eta[\vec{x}:=\vec{d}], \rho[X:=q]}$ is a contractive endofunction on Pred $^{V a l^{n}}$. In particular, the fixed point in the definition of $\llbracket(\mu X(\vec{x}) . P)(\vec{e}) \rrbracket$ is well-defined, and by Lemma B.3,

$$
\llbracket(\mu X(\vec{x}) . P)(\vec{e}) \rrbracket_{\eta}=\lambda \rho \cdot\left(f i x\left(\lambda q, \vec{d} \cdot \llbracket P \rrbracket_{\eta[\vec{x}:=\vec{d}], \rho[X:=q]}\right)\right)\left(\llbracket \vec{e} \rrbracket_{\eta}\right)
$$

is a non-expansive function.

Similarly, if $P$ is formally contractive in $Y \neq X$, then $\lambda q \cdot \llbracket(\mu X(\vec{x}) \cdot P)(\vec{e}) \rrbracket_{\eta, \rho[Y:=q]}$ is contractive by Lemma $\mathrm{B} .3$ and the inductive hypothesis that $q \mapsto \llbracket P \rrbracket_{\eta, \rho^{\prime}[Y:=q]}$ is contractive for any $\rho^{\prime}$.

- It remains to consider the case of (nested) triples. Note that the interpretation of triples is defined in terms of the admissible downward closure, so it is clear that $\llbracket\{P\} e\{Q\} \rrbracket_{\eta, \rho} w$ is uniform and admissible. We first prove claim (1), i.e., the non-expansiveness of $\llbracket\left\{P_{1}\right\} e\left\{Q_{1}\right\} \rrbracket_{\eta, \rho}$. To this end, assume that $w \stackrel{n}{=} w^{\prime}$, and let $h \in \llbracket\{P\} e\{Q\} \rrbracket_{\eta, \rho} w$. We must show that $\pi_{n}(h) \in \llbracket\{P\} e\{Q\} \rrbracket_{\eta} w^{\prime}$. By the downward closure, we also know that $\pi_{n}(h) \in \llbracket\{P\} e\{Q\} \rrbracket_{\eta, \rho} w$. Since $k \stackrel{\text { def }}{=} r n k\left(\pi_{n}(h)\right) \leq n$, we also have $w \stackrel{k}{=} w^{\prime}$. Without loss of generality we can assume that $k>0$, and thus must have $w \models_{k-1}$ $\left\{\llbracket P \rrbracket_{\eta, \rho}\right\} \llbracket e \rrbracket_{\eta}\left\{\llbracket Q \rrbracket_{\eta, \rho}\right\}$. By Lemma 4.8 this implies $w^{\prime} \models_{k-1}\left\{\llbracket P \rrbracket_{\eta, \rho}\right\} \llbracket e \rrbracket_{\eta}\left\{\llbracket Q \rrbracket_{\eta, \rho}\right\}$, and thus also $\pi_{n}(h) \in \llbracket\{P\}$ e $\{Q\} \rrbracket_{\eta, \rho}$.

We now prove the following claim which implies the non-expansiveness and contractiveness properties stated in conditions (2) and (3):

$$
\rho \stackrel{n}{=} \rho^{\prime} \Rightarrow \llbracket\{P\} e\{Q\} \rrbracket_{\eta, \rho} \stackrel{n+1}{=} \llbracket\{P\} \text { e }\{Q\} \rrbracket_{\eta, \rho^{\prime}}
$$

For the proof of this claim, assume $\rho \stackrel{n}{=} \rho^{\prime}$ and $h \in \llbracket\{P\} e\{Q\} \rrbracket_{\eta, \rho} w$ for some $w$. We must show that $\pi_{n+1}(h) \in \llbracket\{P\} e\{Q\} \rrbracket_{\eta, \rho^{\prime}} w$. Let $k \stackrel{\text { def }}{=} r n k\left(\pi_{n+1}(h)\right) \leq n+1$. Without loss of generality we can assume $k>0$ (and hence $k-1 \leq n$ ), and thus obtain $w \models_{k-1}\left\{\llbracket P \rrbracket_{\eta, \rho}\right\} \llbracket e \rrbracket_{\eta}\left\{\llbracket Q \rrbracket_{\eta, \rho}\right\}$. By induction hypothesis, $\llbracket P \rrbracket_{\eta}$ and $\llbracket Q \rrbracket_{\eta}$ are nonexpansive, and thus $\llbracket P \rrbracket_{\eta, \rho} \stackrel{k=1}{=} \llbracket P \rrbracket_{\eta, \rho^{\prime}}$ and $\llbracket Q \rrbracket_{\eta, \rho} \stackrel{k=1}{=} \llbracket Q \rrbracket_{\eta, \rho^{\prime}}$. By Lemma 4.8 we obtain $w \models_{k-1}\left\{\llbracket P \rrbracket_{\eta, \rho^{\prime}}\right\} \llbracket e \rrbracket_{\eta}\left\{\llbracket Q \rrbracket_{\eta, \rho^{\prime}}\right\}$. This yields $\pi_{n+1}(h) \in \llbracket\{P\} e\{Q\} \rrbracket_{\eta, \rho^{\prime}} w$. 
B.3. Soundness of standard rules from separation logic. The following lemmas show that the usual rules of separation logic, expressed using triples containing quoted commands as shown in Figure 3, are sound.

Lemma B.5 (Skip). The axiom $\{P\}$ 'skip' $\{P\}$ is valid.

Proof. This follows from the fact that $\llbracket$ skip $\rrbracket_{\eta} h=h$ for all $h \in$ Heap, and that $\operatorname{Ad}(\cdot)$ is a closure operation.

Lemma B.6 (Conditional). If $\left\{P \wedge e_{0}=e_{1}\right\}$ ' $C$ ' $\{Q\}$ and $\left\{P \wedge e_{0} \neq e_{1}\right\}$ ' $D$ ' $\{Q\}$ are both valid, then so is $\{P\}$ 'if $\left(e_{0}=e_{1}\right)$ then $C$ else $D$ ' $\{Q\}$.

Proof. Let $w \in W$ and $r \in U A d m$ and suppose $h \in \llbracket P \rrbracket_{\eta, \rho} w * \iota^{-1}(w)(e m p) * r$. From the semantics of the conditional, we can assume without loss of generality that $\llbracket e_{0} \rrbracket_{\eta}$ and $\llbracket e_{1} \rrbracket_{\eta}$ are not both in $C o m_{\perp}$. We must show that

$$
c(h) \in \operatorname{Ad}\left(\llbracket Q \rrbracket_{\eta, \rho} w * \iota^{-1}(w)(e m p) * r\right),
$$

where $c(h)=$ if $\left(\llbracket e_{0} \rrbracket_{\eta}=\llbracket e_{1} \rrbracket_{\eta}\right)$ then $\llbracket C \rrbracket_{\eta} h$ else $\llbracket D \rrbracket_{\eta} h$. Depending on whether the statement $\llbracket e_{0} \rrbracket_{\eta}=\llbracket e_{1} \rrbracket_{\eta}$ hold, we have $\llbracket e_{0}=e_{1} \rrbracket_{\eta} w=$ Heap or $\llbracket e_{0} \neq e_{1} \rrbracket_{\eta} w=$ Heap. Therefore, the claim follows from either the first or the second assumed triple.

Lemma B.7 (Update). The axiom $\left\{e \mapsto_{-} * P\right\}$ ' $[e]:=e_{0}{ }^{\prime}\left\{e \mapsto e_{0} * P\right\}$ is valid.

Proof. By Lemma 4.15, it suffices to prove the validity of

$$
\left\{e \mapsto \mapsto_{-}\right\}^{\prime}[e]:=e_{0}{ }^{\prime}\left\{e \mapsto e_{0}\right\} .
$$

Let $\eta \in E n v, \rho \in \operatorname{Pred}^{\Xi}, p=\llbracket e \mapsto-\rrbracket_{\eta, \rho}, q=\llbracket e \mapsto e_{0} \rrbracket_{\eta, \rho}$ and $c=\llbracket[e]:=e_{0} \rrbracket_{\eta}$. We will show that $w \models\{p\} c\{q\}$ holds for all $w \in W$.

Let $w \in W$ and $r \in U A d m$, and suppose $h \in p(w) * \iota^{-1}(w)(e m p) * r$. We may assume that $h \neq \perp$, for otherwise $c(h)=\perp \in q(w) * \iota^{-1}(w)(e m p) * r$ is immediate. Thus, $h=h^{\prime} \cdot h^{\prime \prime}$ such that $h^{\prime} \in p(w)$ and $h^{\prime \prime} \in \iota^{-1}(w)(e m p) * r$. In particular, since $h^{\prime} \in p(w)=\llbracket e \mapsto_{-} \rrbracket_{\eta, \rho} w$, we obtain that $\llbracket e \rrbracket_{\eta} \in \operatorname{dom}\left(h^{\prime}\right) \subseteq \operatorname{dom}(h)$. Therefore, from the semantics of the assignment command, $c(h)=h\left[\llbracket e \rrbracket_{\eta} \mapsto \llbracket e_{0} \rrbracket_{\eta}\right]$. But this heap is the same as $\left\{\llbracket \llbracket e \rrbracket_{\eta}=\llbracket e_{1} \rrbracket_{\eta}\right\} \cdot h^{\prime \prime}$, and therefore $c(h) \in q(w) * \iota^{-1}(w)(e m p) * r$. The latter set is contained in $\operatorname{Ad}\left(q(w) * \iota^{-1}(w)(e m p) *\right.$ $r)$ since $\operatorname{Ad}(\cdot)$ is a closure operation.

Lemma B.8 (UpdateInv). The axiom

UPDATEINV

$$
\left.\overline{\Xi ; \Gamma \vdash\left\{e \mapsto \_*\left(e_{1} \mapsto e_{0} \wedge \phi\right)\right\}^{\prime}[e]:=e_{0}{ }^{\prime}\left\{\left(e \mapsto e_{0} \wedge \phi\right) *\left(e_{1} \mapsto e_{0} \wedge \phi\right)\right\}} \quad \text { ( } \phi \text { pseudo pure }\right)
$$

is valid.

Proof. Consider $\eta \in E n v, \rho \in \operatorname{Pred}^{\Xi}, c=\llbracket[e]:=e_{0} \rrbracket_{\eta}, p=\llbracket e \mapsto-* e_{1} \mapsto e_{0} \wedge \phi \rrbracket_{\eta, \rho}$ and $q=\llbracket\left(e \mapsto e_{0} \wedge \phi\right) *\left(e_{1} \mapsto e_{0} \wedge \phi\right) \rrbracket_{\eta, \rho}$. We will show that $w \models\{p\} c\{q\}$ holds for all $w \in W$.

Let $w \in W$ and $r \in U A d m$, and suppose $h \in p(w) * \iota^{-1}(w)(e m p) * r$. We may assume that $h \neq \perp$, for otherwise $c(h)=\perp \in q(w) * \iota^{-1}(w)(e m p) * r$ is immediate. Thus, $h=h^{\prime} \cdot h^{\prime \prime}$ such that $h^{\prime} \in p(w)$ and $h^{\prime \prime} \in \iota^{-1}(w)(e m p) * r$. In particular, since $h^{\prime} \in$ $p(w)=\llbracket e \mapsto \mapsto_{-} *\left(e_{1} \mapsto e_{0} \wedge \phi\right) \rrbracket_{\eta, \rho} w$, we obtain that $h^{\prime}=h_{1} \cdot h_{2}$ such that $\left\{\llbracket e \rrbracket_{\eta}\right\}=\operatorname{dom}\left(h_{1}\right) \subseteq$ 
$\operatorname{dom}\left(h^{\prime}\right) \subseteq \operatorname{dom}(h)$ and $\left\{\llbracket e_{1} \rrbracket_{\eta}\right\}=\operatorname{dom}\left(h_{2}\right) \subseteq \operatorname{dom}\left(h^{\prime}\right) \subseteq \operatorname{dom}(h)$ and $h_{2} \in \llbracket \phi \rrbracket_{\eta, \rho} w$. Therefore, from the semantics of the assignment command, $c(h)=h\left[\llbracket e \rrbracket_{\eta} \mapsto \llbracket e_{0} \rrbracket_{\eta}\right]$. But this heap is the same as $\left(\left\{\left|\llbracket e \rrbracket_{\eta}=\llbracket e_{0} \rrbracket_{\eta}\right|\right\} \cdot\left\{\left|\llbracket e_{1} \rrbracket_{\eta}=\llbracket e_{0} \rrbracket_{\eta}\right|\right\}\right) \cdot h_{2}$. Now the rank of heap $\left\{\mid \llbracket e \rrbracket_{\eta}=\llbracket e_{0} \rrbracket_{\eta}\right\}$ is obviously identical to the rank of $\left\{\llbracket \llbracket e_{1} \rrbracket_{\eta}=\llbracket e_{0} \rrbracket_{\eta} \mid\right\}$ and thus $\left\{\llbracket \llbracket e \rrbracket_{\eta}=\llbracket e_{0} \rrbracket_{\eta} \mid\right\} \in \llbracket \phi \rrbracket_{\eta, \rho}$ as $\phi$ is pseudo pure and $\left\{\llbracket \llbracket e_{1} \rrbracket_{\eta}=\llbracket e_{0} \rrbracket_{\eta}\right\}=h_{2} \in \llbracket \phi \rrbracket_{\eta, \rho} w$. Therefore $c(h) \in q(w) * \iota^{-1}(w)(e m p) * r$. The latter set is contained in $\operatorname{Ad}\left(q(w) * \iota^{-1}(w)(e m p) * r\right)$ since $\operatorname{Ad}(\cdot)$ is a closure operation.

Lemma B.9 (Free). The axiom $\left\{e \mapsto_{-} * P\right\}$ ' $\mathrm{free}(e)$ ' $\{P\}$ is valid.

Proof. By Lemma 4.15, it suffices to prove the validity of

$$
\left\{e \mapsto_{-}\right\} \text {'free }(e)^{\prime}\{e m p\} .
$$

Let $\eta \in E n v, \rho \in \operatorname{Pred}^{\Xi}, p=\llbracket e \mapsto \rrbracket_{\eta, \rho}, q=\llbracket e m p \rrbracket_{\eta, \rho}$ and $c=\llbracket$ free $(e) \rrbracket_{\eta}$. We will prove that $w \models\{p\} c\{q\}$ holds for all $w \in W$.

Let $w \in W$, let $r \in U A d m$ and suppose $h \in p(w) * \iota^{-1}(w)(e m p) * r$. Since $q(w)$ is the unit for $*$ and $\operatorname{Ad}(\cdot)$ is a closure operation, we must only show $c(h) \in \iota^{-1}(w)(e m p) * r$. We may assume that $h \neq \perp$, for otherwise $c(h)=\perp \in \iota^{-1}(w)(e m p) * r$ is immediate. Thus, $h=h^{\prime} \cdot h^{\prime \prime}$ such that $h^{\prime} \in p(w)$ and $h^{\prime \prime} \in \iota^{-1}(w)(e m p) * r$. In particular, since $h^{\prime} \in p(w)=\llbracket e \mapsto-\rrbracket_{\eta, \rho} w$, we obtain that $\left\{\llbracket e \rrbracket_{\eta}\right\}=\operatorname{dom}\left(h^{\prime}\right) \subseteq \operatorname{dom}(h)$. Therefore, from the semantics of the deallocation command, $c(h)=h^{\prime \prime}$. It follows that $c(h) \in \iota^{-1}(w)(e m p) * r$.

Lemma B.10 (Deref). If $\{P * e \mapsto x\}^{\prime} C$ ' $\{Q\}$ is valid and $x$ is not free in $e$ and $Q$, then $\{\exists x . P * e \mapsto x\}$ 'let $x=[e]$ in $C^{\prime}\{Q\}$ is also valid.

Proof. Assume that $\{P * e \mapsto x\}^{\prime} C$ ' $\{Q\}$ is valid, and pick $\eta \in E n v$ and $\rho \in \operatorname{Pred}^{\Xi}$. Let $c=\llbracket$ let $x=[e]$ in $C \rrbracket_{\eta}$. We will show that $w \models\left\{\llbracket \exists x . P * e \mapsto x \rrbracket_{\eta, \rho}\right\} c\left\{\llbracket Q \rrbracket_{\eta, \rho}\right\}$ for all $w \in W$.

Let $w \in W, r \in U A d m$ and $h \in \llbracket \exists x . P * e \mapsto x \rrbracket_{\eta, \rho}(w) * \iota^{-1}(w)(e m p) * r$. We must show that $c(h) \in \operatorname{Ad}\left(\llbracket Q \rrbracket_{\eta, \rho}(w) * \iota^{-1}(w)(e m p) * r\right)$. By definition there are heaps $h^{\prime}, h^{\prime \prime}$ such that $h=h^{\prime} \cdot h^{\prime \prime}$ and $h^{\prime} \in \llbracket \exists x . P * e \mapsto x \rrbracket_{\eta, \rho}(w)$ and $h^{\prime \prime} \in \iota^{-1}(w)(e m p) * r$. By definition this means that

$$
\forall n . \exists d_{n} \in \text { Val. } \pi_{n}\left(h^{\prime}\right) \in \llbracket P * e \mapsto x \rrbracket_{\eta\left[x:=d_{n}\right], \rho}(w) .
$$

Let us write $\eta_{n}$ for $\eta\left[x:=d_{n}\right]$. In the remainder of the proof, we will prove that

$$
\forall n . c\left(\pi_{n}(h)\right) \in \operatorname{Ad}\left(\llbracket Q \rrbracket_{\eta, \rho} * \iota^{-1}(w)(e m p) * r\right),
$$

because then, by admissibility and the continuity of $c$, we obtain the required $c(h) \in$ $\operatorname{Ad}\left(\llbracket Q \rrbracket_{\eta, \rho} * \iota^{-1}(w)(e m p) * r\right)$.

Without loss of generality we can assume that $\pi_{n}(h) \neq \perp$, so that $\pi_{n}\left(h^{\prime}\right) \neq \perp$ as well. Then, since $x \notin f v(e)$, we have in particular $\llbracket e \rrbracket_{\eta} \in \operatorname{dom}\left(\pi_{n}\left(h^{\prime}\right)\right) \subseteq \operatorname{dom}(h)$ and $\pi_{n}\left(h^{\prime}\right)\left(\llbracket e \rrbracket_{\eta}\right) \sqsubseteq d_{n}$. Using the monotonicity of commands with respect to the environment, this gives

$$
c\left(\pi_{n}(h)\right)=\llbracket C \rrbracket_{\eta\left[x:=\pi_{n}\left(h^{\prime}\right)\left(\llbracket e \rrbracket_{\eta}\right)\right]}\left(\pi_{n}(h)\right) \sqsubseteq \llbracket C \rrbracket_{\eta_{n}}\left(\pi_{n}(h)\right)
$$

By uniformity of $\iota^{-1}(w)(e m p) * r$, we have $\pi_{n}(h) \in \llbracket P * e \mapsto x \rrbracket_{\eta_{n}, \rho} * \iota^{-1}(w)(e m p) * r$, so that the assumption gives us

$$
c\left(\pi_{n}(h)\right) \sqsubseteq \llbracket C \rrbracket_{\eta_{n}}\left(\pi_{n}(h)\right) \in \operatorname{Ad}\left(\llbracket Q \rrbracket_{\eta_{n}, \rho} * \iota^{-1}(w)(e m p) * r\right) .
$$

Since $\operatorname{Ad}\left(p^{\prime}\right)$ is a downward-closed set for every predicate $p^{\prime}$, the above formula implies that $c\left(\pi_{n}(h)\right)$ belongs to the set on the right hand side. Furthermore, since $x \notin f v(Q)$, 
we have $\llbracket Q \rrbracket_{\eta_{n}}=\llbracket Q \rrbracket_{\eta}$. The combination of these two facts gives the desired $c\left(\pi_{n}(h)\right) \in$ $\operatorname{Ad}\left(\llbracket Q \rrbracket_{\eta, \rho} * \iota^{-1}(w)(e m p) * r\right)$.

Lemma B.11 (New). If $\{P * x \mapsto e\}$ ' $C$ ' $\{Q\}$ is valid and $x$ is not free in $P, Q$ and $e$, then $\{P\}$ 'let $x=$ new $e$ in $C$ ' $\{Q\}$ is valid.

Proof. Let $w \in W, \eta \in E n v, \rho \in \operatorname{Pred}^{\Xi}$ and $r \in U A d m$. Suppose $h \in \llbracket P \rrbracket_{\eta, \rho}(w) *$ $\iota^{-1}(w)(e m p) * r$. We must show that $c(h) \in \operatorname{Ad}\left(\llbracket Q \rrbracket_{\eta, \rho}(w) * \iota^{-1}(w)(e m p) * r\right)$. Consider the following environment $\eta^{\prime}$ and heap $h^{\prime}$ :

$$
\eta^{\prime} \stackrel{\text { def }}{=} \eta[x:=\ell] \quad \quad h^{\prime} \stackrel{\text { def }}{=} h \cdot\left\{\mid \ell=\llbracket e \rrbracket_{\eta}\right\}
$$

where $\ell$ is the least natural number not contained in $\operatorname{dom}(h)$. Since $x$ is not free in $e$ and $P$, we have $\llbracket e \rrbracket_{\eta}=\llbracket e \rrbracket_{\eta^{\prime}}$ and $\llbracket P \rrbracket_{\eta}=\llbracket P \rrbracket_{\eta^{\prime}}$. Thus by the assumption on $h$ we obtain:

$$
h^{\prime} \in \llbracket P * x \mapsto e \rrbracket_{\eta^{\prime}, \rho} w * \iota^{-1}(w)(e m p) * r .
$$

Then the assumption that $\{P * x \mapsto e\}$ ' $C$ ' $\{Q\}$ is valid implies:

$$
\llbracket C \rrbracket_{\eta^{\prime}} h^{\prime} \in \operatorname{Ad}\left(\llbracket Q \rrbracket_{\eta^{\prime}, \rho}(w) * \iota^{-1}(w)(e m p) * r\right) .
$$

Using the fact that $\llbracket$ let $x=$ new $e$ in $C \rrbracket_{\eta}(h)=\llbracket C \rrbracket_{\eta^{\prime}} h^{\prime}$ and since $\llbracket Q \rrbracket_{\eta^{\prime}}=\llbracket Q \rrbracket_{\eta}$, this proves the statement.

Lemma B.12 (Auxiliary variable). Assume that $x$ is not free in $e$. Then the axiom

ExIsTAux

$$
\overline{\Gamma \vdash(\forall x .\{P\} e\{Q\}) \Rightarrow\{\exists x . P\} e\{\exists x \cdot Q\}}
$$

is valid.

Proof. Let $\eta \in$ Env, $\rho \in \operatorname{Pred}^{\Xi}$, and fix $w \in W$. For each $d \in V a l$, let $\eta_{d}=\eta[x:=d]$, $p_{d}=\llbracket P \rrbracket_{\eta_{d}, \rho}$ and $q_{d}=\llbracket Q \rrbracket_{\eta_{d}, \rho}$. Since $x$ is not free in $e$, we have $\llbracket e \rrbracket_{\eta_{d}}=\llbracket e \rrbracket_{\eta}$. Thus, a similar reasoning with rank as that in the proof of Consequence implies that it is sufficient to prove the following claim:

for all $c$, if $w \models\left\{p_{d}\right\} c\left\{q_{d}\right\}$ for every $d$, then $w \models\left\{\bigsqcup_{d} p_{d}\right\} c\left\{\bigsqcup_{d} q_{d}\right\}$.

Assume $w \models\left\{p_{d}\right\} c\left\{q_{d}\right\}$, let $r \in U A d m$ and $h \in\left(\bigsqcup_{d} p_{d}\right)(w) * \iota^{-1}(w)(e m p) * r$. We must show that $c(h) \in \operatorname{Ad}\left(\left(\bigsqcup_{d} q_{d}\right)(w) * \iota^{-1}(w)(e m p) * r\right)$. By definition, $h=h^{\prime} \cdot h^{\prime \prime}$ where $h^{\prime} \in\left(\bigsqcup_{d} q_{d}\right)(w)$ and $h^{\prime \prime} \in \iota^{-1}(w)(e m p) * r$. Thus, for each $n$ there exists $d \in V a l$ such that $\pi_{n}\left(h^{\prime}\right) \in p_{d}(w)$, and therefore $\pi_{n}(h) \in p_{d}(w) * \iota^{-1}(w)(e m p) * r$ by the uniformity of $\iota^{-1}(w)(e m p) * r$. From the assumption $w \models\left\{p_{d}\right\} c\left\{q_{d}\right\}$ we then obtain that for each $n$,

$$
c\left(\pi_{n}(h)\right) \in \operatorname{Ad}\left(q_{d}(w) * \iota^{-1}(w)(e m p) * r\right) \subseteq \operatorname{Ad}\left(\left(\bigsqcup_{d} q_{d}\right)(w) * \iota^{-1}(w)(e m p) * r\right) .
$$

Using the admissibility of $\operatorname{Ad}\left(\left(\bigsqcup_{d} q_{d}\right)(w) * \iota^{-1}(w)(e m p) * r\right)$ and the continuity of $c$, it follows that $c(h) \in \operatorname{Ad}\left(\left(\bigsqcup_{d} q_{d}\right)(w) * \iota^{-1}(w)(e m p) * r\right)$. 
Lemma B.13 (Invariance). Then the axiom

$$
\begin{aligned}
& \text { INVARIANCE } \\
& \Xi ; \Gamma \vdash\{P\} e\{Q\} \Rightarrow\{P \wedge \psi\} e\{Q \wedge \psi\} \quad(\psi \text { is pure })
\end{aligned}
$$

is valid.

Proof. Let $\eta \in E n v, \rho \in \operatorname{Pred}^{\Xi}$, and fix $w \in W$. For each $d \in V a l$,let $p=\llbracket P \rrbracket_{\eta, \rho}$ and $q=\llbracket Q \rrbracket_{\eta, \rho}$ and $f=\llbracket \psi \rrbracket_{\eta, \rho}$. A similar reasoning with rank as that in the proof of (CONSEQ) implies that it is sufficient to prove the following claim:

$$
\text { for all } c \text {, if } w \models\{p\} c\{q\} \text { then } w \models\{p \cap f\} c\{q \cap f\} \text {. }
$$

But since $\psi$ is pure, either $f w=$ Heap for all $w \in W$ or $f w=\emptyset$ for all $w \in W$. In the former case, the above implication reduces to the identity axiom, in the latter case $w \models\{p \cap f\} c\{q \cap f\}$ always holds.

Lemma B.14 (Disjunction). For all $P, P^{\prime}, Q, Q^{\prime}$ and $e$, the axiom

DisJ

is valid.

$$
\overline{\{P\} e\{Q\} \wedge\left\{P^{\prime}\right\} e\left\{Q^{\prime}\right\} \Rightarrow\left\{P \vee P^{\prime}\right\} e\left\{Q \vee Q^{\prime}\right\}}
$$

Proof. Let $\eta \in E n v, \rho \in \operatorname{Pred}^{\Xi}$, and fix $w \in W$. Let $p=\llbracket P \rrbracket_{\eta, \rho}, p^{\prime}=\llbracket P^{\prime} \rrbracket_{\eta, \rho}, q=\llbracket Q \rrbracket_{\eta, \rho}$ and $\llbracket Q^{\prime} \rrbracket_{\eta, \rho}$. As in the preceding proofs, it suffices to show that

$$
\text { for all } c \text {, if } w \models\{p\} c\{q\} \text { and } w \models\left\{p^{\prime}\right\} c\left\{q^{\prime}\right\} \text {, then } w \models\left\{p \cup p^{\prime}\right\} c\left\{q \cup q^{\prime}\right\} \text {. }
$$

For this, suppose that $r \in U A d m$ and let $h \in\left(p \cup p^{\prime}\right)(w) * \iota^{-1}(w)(e m p) * r$. We must show that $c(h) \in\left(q \cup q^{\prime}\right)(w) * \iota^{-1}(w)(e m p) * r$. Note that $h \in\left(p \cup p^{\prime}\right)(w) * \iota^{-1}(w)(e m p) * r$ entails that $h \in p(w) * \iota^{-1}(w)(e m p) * r$ or $h \in p^{\prime}(w) * \iota^{-1}(w)(e m p) * r$. Therefore, by the assumption we know that $c(h) \in \operatorname{Ad}\left(q(w) * \iota^{-1}(w)(e m p) * r\right)$ or $c(h) \in \operatorname{Ad}\left(q^{\prime}(w) * \iota^{-1}(w)(e m p) * r\right)$, from which it follows that $c(h) \in \operatorname{Ad}\left(\left(q \cup q^{\prime}\right)(w) * \iota^{-1}(w)(e m p) * r\right)$ by the monotonicity of $*$ and of the closure operation. 